\title{
Volatile Organic Compound Profiles From Wheat Diseases Are Pathogen-Specific and Can Be Exploited for Disease Classification
}

\section{OPEN ACCESS}

Edited by:

Tofazzal Islam,

Bangabandhu Sheikh Mujibur Rahman Agricultural University,

Bangladesh

Reviewed by:

Valeria Scala,

Centro di Ricerca Difesa e Sperimentazione (CREA-DC), Italy

Mara Quaglia,

University of Perugia, Italy

*Correspondence: Andrea Ficke andrea.ficke@nibio.no

Specialty section:

This article was submitted to Microbe and Virus Interactions with

Plants,

a section of the journal

Frontiers in Microbiology

Received: 27 October 2021

Accepted: 01 December 2021

Published: 07 January 2022

Citation:

Ficke A, Asalf B and Norli HR (2022) Volatile Organic Compound Profiles From Wheat Diseases Are

Pathogen-Specific and Can Be Exploited for Disease Classification.

Front. Microbiol. 12:803352. doi: 10.3389/fmicb.2021.803352

\author{
Andrea Ficke*, Belachew Asalf and Hans Ragnar Norli \\ Division of Biotechnology and Plant Health, Norwegian Institute of Bioeconomy Research (NIBIO), Ås, Norway
}

Plants and fungi emit volatile organic compounds (VOCs) that are either constitutively produced or are produced in response to changes in their physico-chemical status. We hypothesized that these chemical signals could be utilized as diagnostic tools for plant diseases. VOCs from several common wheat pathogens in pure culture (Fusarium graminearum, Fusarium culmorum, Fusarium avenaceum, Fusarium poae, and Parastagonospora nodorum) were collected and compared among isolates of the same fungus, between pathogens from different species, and between pathogens causing different disease groups [Fusarium head blight (FHB) and Septoria nodorum blotch (SNB)]. In addition, we inoculated two wheat varieties with either F. graminearum or $P$. nodorum, while one variety was also inoculated with Blumeria graminis f.sp. tritici (powdery mildew, PM). VOCs were collected 7, 14, and 21 days after inoculation. Each fungal species in pure culture emitted a different VOC blend, and each isolate could be classified into its respective disease group based on VOCs with an accuracy of 71.4 and $84.2 \%$ for FHB and SNB, respectively. When all collection times were combined, the classification of the tested diseases was correct in 84 and $86 \%$ of all cases evaluated. Germacrene D and sativene, which were associated with FHB infection, and mellein and heptadecanone, which were associated with SNB infection, were consistently emitted by both wheat varieties. Wheat plants infected with PM emitted significant amounts of 1-octen-3-ol and 3,5,5-trimethyl-2-hexene. Our study suggests that VOC blends could be used to classify wheat diseases. This is the first step toward a real-time disease detection in the field based on chemical signatures of wheat diseases.

Keywords: wheat, disease, head space (HS)-GC/MS, volatiles, fungi, sensor, identificatication, classification

\section{INTRODUCTION}

As part of their metabolism, plants and fungi release diverse volatile organic compounds (VOCs) into the air. At the global level, plants alone account for $67 \%$ of the VOC emissions present in the atmosphere (Guenther, 1997; Sankaran et al., 2010). Plant VOCs are emitted in response to biotic and abiotic stresses and play an important role in defense signaling and communication between plants (Brilli et al., 2019). They can serve as priming agents to enhance resistance to both herbivores (Kim and Felton, 2013) and plant pathogens (Ameye et al., 2015) and can serve as important 
stimuli in the stress memory of plants. Plant VOCs can affect hormone levels and are implicated in regulating the senescence processes of competitive plants, such as weeds [reviewed in Arimura et al. (2010)]. Rhythmic release of some flower volatiles appears to be under circadian control (Fenske and Imaizumi, 2016), and the release of VOCs from oak branches fluctuated not only with light and temperature but also with the season and time of day (Pio et al., 2005). About 250 fungal-specific VOCs have been shown to have characteristic odors (Morath et al., 2012). The rate of emission of VOCs from plants and fungi varies depending on the physico-chemical and biological factors and the nature of the interaction between the pathogen and the plant (Sankaran et al., 2010; Morath et al., 2012). However, many VOCs emitted from plants in response to biotic stress factors are nonspecific, as different pathogens can cause similar tissue damage and elicit the same chemical response (Jansen et al., 2011). VOC emissions have been used in pest management strategies such as pheromone-based insect control (Witzgall et al., 2010) and plant volatile-based attract-and-kill management strategies (Gregg et al., 2018). Fungal VOCs have been used as attractants and deterrents to insects and other invertebrates (Morath et al., 2012) and as resistance inducers (Naznin et al., 2013, 2014), but not as biomarkers for disease detection in the field.

Crop plants are known to emit disease-specific VOCs after pathogen infection (Shulaev et al., 1997; Cardoza et al., 2002; Algarra et al., 2015). A recent study from Thompson et al. (2021) found that Rhododendron plants infected with the oomycete Phytophthora ramorum had a distinctly different VOC profile than Rhododendron plants inoculated with Phytophthora cactorum, Rhizoctonia solani, and non- or mock-inoculated plants both in the growth chamber and in the field. These disease-specific VOCs could supply us with information about the pathogen's identity and site of attack (Jansen et al., 2011) and help us to monitor diseases in real time for site-specific control.

The choice of method for collection of VOCs depends on the dynamic, type, and amounts of VOCs expected to be produced. Dynamic headspace sampling of VOCs is a frequently used method for plant VOC sampling and is characterized by a continuous stream of filtered air within a sampling container made of mostly inert, non-bleeding material, such as glass (Stewart-Jones and Poppy, 2006; Tholl et al., 2006). VOCs are captured and enriched on a separate adsorbent material that is later eluted with an organic solvent or desorbed thermally (Tholl et al., 2006). Abel et al. (2009) showed that VOC recovery rates of different target compounds could vary strongly, even if the same collection and elution method was used. Practical application of VOC detection demands portable and highly sensitive sensors. Recent advancement in this field led to the development of miniature gas-chromatography-based samplers and detector, which would facilitate the exploitation of VOCs for disease monitoring (Tholl et al., 2021).

Common wheat (Triticum aestivum L.) is one of the most important staple food crops in the world (Food and Agriculture Organization [FAO], 2019) and subject to several yield-reducing diseases, including Fusarium head blight (FHB), Septoria nodorum blotch (SNB), and powdery mildew (PM; Bockus et al., 2010). The potential yield loss due to pathogens was estimated to be $16 \%$ annually at the global level, increasing with production intensity (Oerke, 2006). Despite the increased use of pesticides over the last 40 years, crop losses have not decreased significantly (Oerke, 2006). This underlines the need for a more effective fungicide application. Broad-field application of fungicides, as currently practiced, applies fungicides to healthy and diseased plants within the field. Fungicides should be applied where disease is actually present. The current practice of spraying fungicides indiscriminately over the whole field leads to redundant use on areas not affected by diseases. Mapping disease hotspots in the field would enable us to apply fungicide in a sitespecific manner, avoiding redundant fungicide use on healthy plants. Accordingly, the application of fungicides guided by pathogen-specific VOCs could significantly reduce the amounts of active compounds needed for disease control in the field (Gebbers and Adamchuk, 2010).

The objective of this manuscript was to determine if VOC profiles are specific for different wheat pathogens and the diseases they are causing in wheat. Accordingly, we addressed these specific hypotheses: (i) Fungal isolates causing diseases in wheat have their own characteristic VOC or VOC blend produced in pure culture. (ii) Plant pathogenic fungal isolates can be grouped into their genera/disease group based on their VOC profiles. (iii) Pathogen VOCs produced in pure culture will also be produced in plants infected with these pathogens. (iv) Infected plants will emit VOC profiles that are specific to a pathogen, allowing correct classification of the different diseases, even when different wheat varieties are used. (v) The release of pathogen-specific VOCs correlates with disease index over time.

\section{MATERIALS AND METHODS}

\section{Fungal and Plant Experiments Fungal Maintenance and Cultivation}

We used the following fungal species and isolates from the NIBIO collection of fungal cereal pathogens in the fungal VOC collection experiment: Parastagonospora nodorum isolates 202586, 201254, 201252, and 201253; Fusarium culmorum isolate 202588; Fusarium avenaceum isolate 202587; Fusarium poae isolate 202589; and Fusarium graminearum isolates 201569, $\mathrm{KISH} 140 / 80$, and KISH61/07. All isolates were stored at $-80^{\circ} \mathrm{C}$ before use. Pathogens were sub-cultured and placed on potato dextrose agar (PDA, Difco ${ }^{\mathrm{TM}}$, Becton, Dickinson and Company, Le Pont de Claix, France) filled in 5-cm Petri dishes and grown for 6-10 days at $20^{\circ} \mathrm{C}$ and 12 -h light/dark periods. Each replicate contained four $5-\mathrm{cm}$ Petri dishes. The diameter of the fungal growth was determined by measuring two lines perpendicular to each other across the Petri dish from one end to the other end of the colony and taking the average of these two measurements.

\section{Inoculum Preparation}

Parastagonospora nodorum (isolate 201254) and F. graminearum (isolate 201569) were grown on PDA in 9-cm Petri dishes placed under near UV light, at 12 -h light/dark periods and $20-25^{\circ} \mathrm{C}$ for 7-10 days to induce sporulation. Spore suspensions were prepared by flooding the sporulating colonies on PDA plates with 
sterile distilled water $(0.1 \%$ Tween 20 , Sigma-Aldrich, Steinheim, Germany). The suspensions were adjusted to $1 \times 10^{6}$ spores $/ \mathrm{ml}$ and filled into handheld pump sprayers for application referred to in the methods of Engle et al. (2003) and Lin et al. (2020). Mixedfield isolates of Blumeria graminis f.sp. tritici were collected from Norwegian spring wheat in 2010, propagated, and maintained on seedlings of spring wheat 'Bjarne' in the greenhouse over the course of the study.

\section{Plant Cultivation and Fungal Inoculation}

Spring wheat varieties 'Bjarne' and 'Zebra' were sown in 11-cm plastic pots (OS Plastic A/S Denmark) filled with the substrate perlite (grade 2, LOG AS, Oslo, Norway). The number of seedlings was thinned down to four to five plants per pot. Plants were grown in the greenhouse $\left(15^{\circ} \mathrm{C} / 18^{\circ} \mathrm{C}\right.$ night/day) under 8 $\mathrm{h}$ dark/16-h light conditions. The plants were regularly fertilized with a balanced nutrient solution and watered. Three pots of each wheat variety were inoculated with an aqueous suspension of $P$. nodorum conidia $\left(10^{6}\right.$ spores $\left./ \mathrm{ml}\right)$ at flag leaf stage $(\mathrm{BBCH}$ 47-49). Another set of three pots per variety was inoculated with an aqueous $F$. graminearum solution $\left(10^{6}\right.$ spores $\left./ \mathrm{ml}\right)$ at flowering time (BBCH 60-65). The control plants (three pots with four to five plants per variety per inoculation time) were only sprayed with distilled water plus Tween 20 (0.1\%). All plants were covered with clear plastic bags for $48 \mathrm{~h}$ after inoculation and kept under the same greenhouse conditions as described before. As 'Zebra' appeared not to be susceptible to our PM field isolate, we inoculated three pots with four to five plants of the susceptible variety 'Bjarne' with B. graminis f.sp. tritici by dusting conidia from young sporulating colonies onto the plants at the threeleaf stage (BBCH 13) using a fine artist's paintbrush (Asalf et al., 2014). For powdery mildew, we used dry inoculum instead of an aqueous conidia suspension, following the method of Asalf et al. (2021). We reduced contamination of the healthy control plants by constructing a closed "mini-greenhouse." The minigreenhouse was constructed from a 1.5-L clear plastic bottle with its bottom removed carefully and placed over the plants in each pot. The bottle top was covered with paper tissue to allow air exchange, while preventing any cross contamination with conidia between the inoculated and control plants.

One pot with inoculated plants and one control pot per variety were chosen 7,14 , and 21 days post inoculation (dpi) for VOC collection from 'Bjarne' infected with $P$. nodorum, F. graminearum, and B. graminis f.sp. tritici. From 'Zebra', we sampled plants inoculated with $P$. nodorum and F. graminearum 7,14 , and $21 \mathrm{dpi}$. We repeated the VOC collections for each pathogen on each of the two varieties two to four times. The 7day sampling time was chosen to confirm successful inoculation and symptom development of all three diseases before VOC collection. The subsequent sampling times of 14 and 21 days allowed us to follow both the increase in disease index and changes in VOC production over time.

\section{Disease Assessments and Plant Biomass Determination}

Disease severity on leaves and stems was visually estimated as percent of plant surface area covered with brown necrotic lesions for SNB or with white mycelium for PM (Zadoks and Schein, 1979). We assessed the overall percentage of infected area from all plants in the pot. The severity of SNB and PM was determined by estimating the percent of leaf and stem area covered with light brown lesions and white powdery mycelium, respectively. The severity of FHB was determined by estimating the percentage of bleached kernels in each head. After disease assessment and VOC collection, all the above-soil parts of the plants were cut off and weighed to determine the fresh weight. The average disease severity was multiplied with the fresh weight of the plant material per pot to determine the disease index (DI) for each disease. This index was developed and used in our study to allow a more comparable disease measurement between plants at different developmental stages following the recommendations by Zadoks and Schein (1979) to define the scale in order to put disease severity into relevant context.

\section{Collection of Volatile Organic Compounds From Pure Fungal Cultures}

Four 5-cm Petri dishes filled with PDA and covered with a specific fungal isolate, 7-10 days old, were placed like a pyramid on top of each other inside a ca. 2.5-L airtight glass container (Figure 1A). PDA plates without fungal growth were used as control. Our experimental set up allowed us to collect VOCs from four different samples at the same time in parallel. We included control samples at each sampling event. We had a total of 50 VOC collections, of which 33 were from fungi (see Table 1, i.e., sum of replicates) and 17 were control. The glass container was equipped with one inlet for charcoal-filtered air and one outlet containing a Super-Q filter (35 mg of 80/100 mesh; Alltech, Deerfield, IL, United States). Charcoal-filtered air was flowing inside the container at $220-240 \mathrm{ml} / \mathrm{min}$ for $24 \mathrm{~h}$ at $18 \pm 2{ }^{\circ} \mathrm{C}$ to collect VOCs from the headspace. The Super-Q filters were covered by aluminum foil to reduce the effect of light on the VOC during the sampling period. The Super-Q filters were cleaned by rinsing sequentially with $6 \mathrm{ml}$ hexane (Sigma-Aldrich, Steinheim, Germany), $6 \mathrm{ml}$ methanol (Sigma-Aldrich, Steinheim, Germany), and $6 \mathrm{ml}$ hexane each time before they were used for VOC collection. The volatile compounds were desorbed from the absorbent by eluting the filter with $0.3 \mathrm{ml}$ hexane, before adding $500 \mathrm{ng}$ heptyl acetate (supplied from SAFC with a purity of $>98 \%$ ) as internal standard to the elutant. The samples were sealed in glass vials and stored at $-80^{\circ} \mathrm{C}$ until further analyses.

All the glass materials used for VOC collection were washed, rinsed by spraying $70 \%$ ethanol, and then heated to $300^{\circ} \mathrm{C}$ for $8 \mathrm{~h}$ before they were reused for VOC collection. We collected VOCs from three different isolates and their control (non-inoculated PDA plates) at the same time.

\section{Collection of Volatile Organic Compounds From Wheat Plants}

The perlite in each pot with wheat plants was covered with aluminum foil to reduce any VOCs emitted from the growth medium and placed into glass chambers of varying sizes, adjusted to the height of the plants. Plants were placed inside the glass chambers, and the chambers were closed airtight with a ground glass fitting (Figure 1B). Depending on the disease 

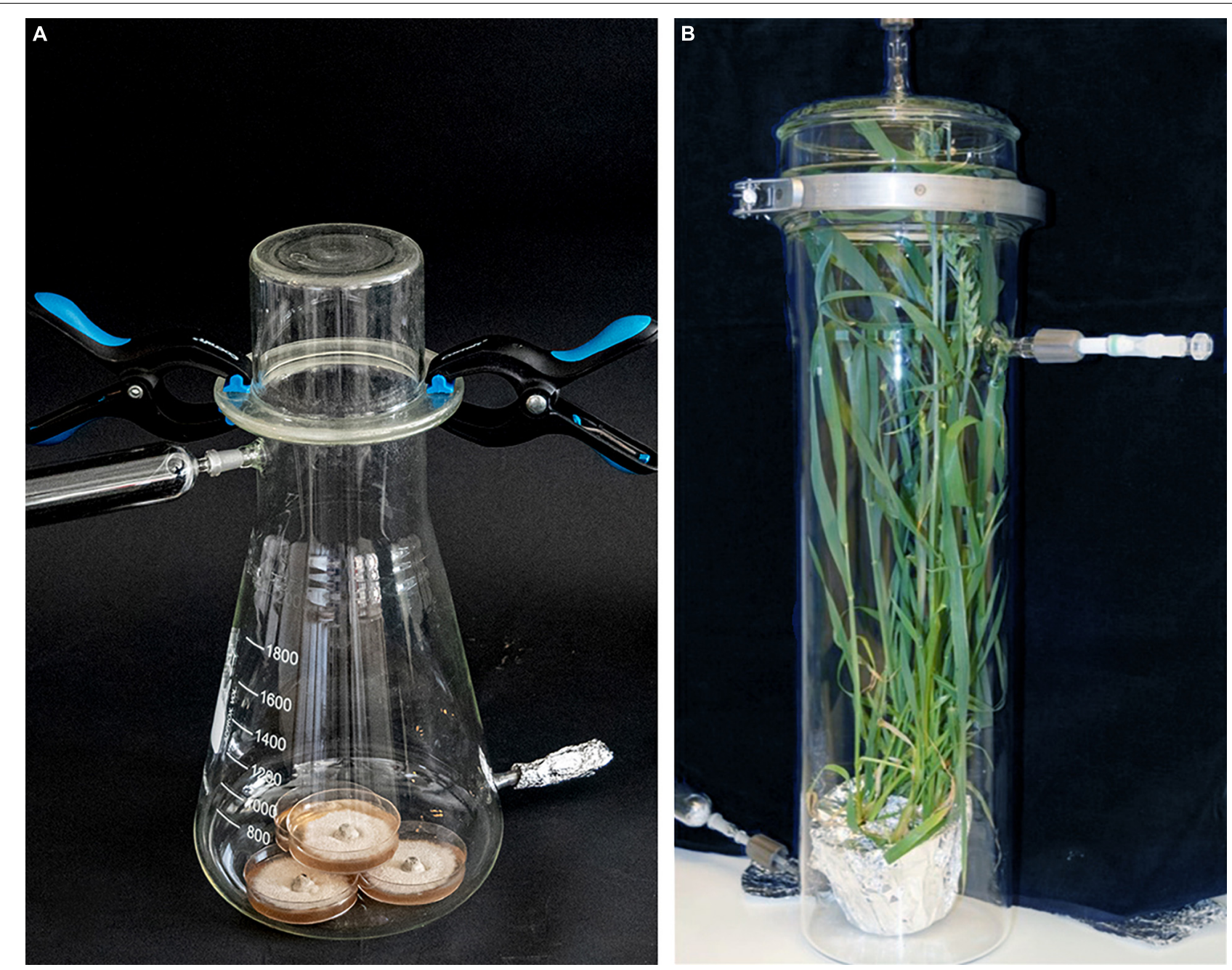

FIGURE 1 | Collection of volatile organic compounds from one fungal isolate on four potato dextrose agar (PDA) plates (5-cm diameter) (A) and from whole wheat plants in an airtight glass container (B). Coal-filtered air is pushed through the glass chamber across the fungal mycelium or the plant surface at $220-240 \mathrm{ml} / \mathrm{min}$, and volatile organic compounds contained in the air flow are collected on Super-Q filter at the other side of the container.

investigated and time after inoculation, the plants were at different developmental stages when placed into the container, and the age of the plant varied somewhat with light conditions and temperature fluctuations in the green house. Wheat plants inoculated with PM at GS 13 and sampled 7, 14, and 21 dpi were approximately 3, 4, and 5 weeks old, respectively. Wheat plants inoculated with SNB at GS 47-49 were approximately 7, 8, and 9 weeks old, respectively, while wheat plants inoculated with FHB at GS 65 were approximately 9, 10, and 11 weeks old, respectively. Charcoal-filtered clean air was pushed through the chamber at a rate of $220-240 \mathrm{ml} / \mathrm{min}$. A Super-Q filter (adsorbent tube) was connected to the chambers' exhaust to collect VOCs from the plant's headspace as described above for $24 \mathrm{~h}$ at $20 \pm 2^{\circ} \mathrm{C}$. The Super-Q filters were covered by aluminum foil during the sampling period as described above. The VOCs were adsorbed, $500 \mathrm{ng}$ of the heptyl acetate standard was added, and the glass vials were stored as described above. We collected VOCs from four glass containers (each containing one pot with plants) at the same time, which included 'Bjarne' and 'Zebra' inoculated with the pathogen and their non-inoculated controls.

\section{Chemical Analysis and Compound Identification}

\section{Gas Chromatography and Mass Spectrometry}

Instrument settings for volatile analysis, including gas chromatography (GC) column type and temperature program together with mass spectrometry (MS) parameters, are described by Dalen et al. (2015).

\section{Peak Selection and Determination of Relative Amount of Volatile Organic Compounds}

The Deconvolution Reporting Software (DRS-version A.02.00, Agilent Technologies) in combination with the Automated Mass Spectral Deconvolution and Identification System (AMDIS) 
TABLE 1 | Number of replications and average sum of colony area with standard error for each fungal isolate grown on four $5-\mathrm{cm}$ Petri plates at time of volatile organic compound collection (6-10 days after transfer).

\begin{tabular}{lccc}
\hline Fungal species & Isolate number & $\begin{array}{c}\text { Number of } \\
\text { replicates }(\boldsymbol{n})^{\mathbf{b}}\end{array}$ & $\begin{array}{c}\text { Sum of colony } \\
\text { area } \mathbf{( c m}^{\mathbf{2}} \mathbf{\text { per }} \\
\text { replicate }^{\mathbf{c}}\end{array}$ \\
\hline Parastagonospora nodorum & 202586 & 3 & $32.57^{\mathrm{BC}}$ \\
Parastagonospora nodorum & $201254^{\mathrm{a}}$ & 4 & $25.73^{\mathrm{C}}$ \\
Parastagonospora nodorum & 201252 & 4 & $29.77 \mathrm{C}$ \\
Parastagonospora nodorum & 201253 & 4 & $43.98^{\mathrm{B}}$ \\
Fusarium culmorum & 202588 & 3 & $78.50^{\mathrm{A}}$ \\
Fusarium avenaceum & 202587 & 3 & $78.50^{\mathrm{A}}$ \\
Fusarium poae & 202589 & 3 & $78.50^{\mathrm{A}}$ \\
Fusarium graminearum & $201569^{\mathrm{a}}$ & 3 & $44.00^{\mathrm{B}}$ \\
Fusarium graminearum & $\mathrm{KISH} 140 / 80$ & 3 & $78.50^{\mathrm{A}}$ \\
Fusarium graminearum & $\mathrm{KISH} 61 / 07$ & 3 & $78.50^{\mathrm{A}}$ \\
\hline
\end{tabular}

a These isolates were also used in the plant inoculation.

${ }^{b}$ A replicate consisted of four Petri plates at each collection time.

${ }^{c}$ Different letters in this column indicate significant differences based on Tukey's test with $5 \%$ error rate.

database $^{1}$ was used for identification of volatile compounds. Details regarding DRS and AMDIS settings are described by Steen et al. (2019). Peaks present in the chromatogram but not identified by the DRS were manually interpreted through the NIST08-database. For reliable identification, a match factor $\geq 70$ was employed (Stein, 1999). Additionally, MetAlign, version 04101 settings as in Steen et al. (2019), was used to investigate if compounds missed by AMDIS or NIST would appear by differential analysis of data sets comparing inoculated and noninoculated PDA Petri dishes and inoculated and control plants (Tikunov et al., 2005). Compounds of interest were selected if they were consistently detected in the fungal samples or inoculated plants. Relative amounts of microgram heptyl acetate equivalents of identified compounds were calculated by dividing the peak area (using the area from the total ion chromatogram) by the area of the internal standard heptyl acetate. The VOCs octanal, 1-octen-3-ol, sativene, 1-octanol, germacrene D, and mellein were verified by comparison with synthetic standards. The compound mellein was provided by Yngve H. Stenstrøm (Norwegian University of Life Sciences, Ås, Norway). All other standards were supplied by Sigma-Aldrich, Fluka, Chiron AS, Supleco, and SAFC with a purity ranging from 95 to $99 \%$.

\section{Statistical Analysis}

\section{Fungal Growth on Potato Dextrose Agar}

We calculated the average sum of the colony areas for each repeated experiment. One experiment consisted of collecting VOCs from three isolates (four 5-cm Petri dishes) and one control (four 5-cm Petri dish only filled with PDA) in parallel. The experiment was repeated three to four times. We compared fungal growth using Tukey's test with an error rate of 5\% assuming equal variance.

\footnotetext{
${ }^{1}$ http://www.amdis.net/
}

\section{Disease Severity, Fresh Biomass, and Disease Index}

The disease severity, biomass, and disease index data taken from inoculated wheat plants were normally distributed, and we calculated the means of the disease severity 7, 14, and $21 \mathrm{dpi}$. We compared disease severity, fresh biomass, and disease index between different collection times $(7,14$, and $21 \mathrm{dpi}$ ) for each variety-pathogen combination using Tukey's test for pairwise comparison with an error rate of 5\% assuming equal variance. There was no disease on plants not inoculated with the different pathogens, and the disease index was therefore zero during each of the collection times for the controls. Data on disease severity, biomass, and disease index were therefore not analyzed statistically for non-inoculated plants. One experiment consisted of one pot inoculated and one pot control 'Zebra' plants and one pot of inoculated and one pot of control 'Bjarne' plants. We repeated the collection of VOCs from 'Zebra' and 'Bjarne' plants three to four times, except for the collection of VOCs from 'Bjarne' plants at $14 \mathrm{dpi}$ with PM, which we repeated two times.

\section{Determining Which Volatile Organic Compounds Were Produced by Fungal Isolates in vitro}

The median amounts for each of the selected VOCs for all replicates were calculated for each isolate. The number of replicates was three for all $F$. graminearum isolates; four for $F$. avenaceum, F. culmorum, and F. poae isolates; and five for all $P$. nodorum isolates, except for $P$. nodorum isolate number 201254, where we repeated the collection four times. We included the control (PDA only) during each collection event (17 control samples in total). The data for the different VOCs collected from inoculated PDA plates were not normally distributed, as there were many zeros in the data set. Transformation of the data did not normalize the data, and we decided to use a non-parametric Kruskal-Wallis test to determine if the median amount of each collected compound differed significantly between isolates and control plates. $P$-values were adjusted for ties, and the significance level was set to $P<0.05$ (Table 2).

\section{Classification of Fungal Cultures Into Their Respective Isolate and Disease Group}

We used a discriminant analysis based on a linear model to group the isolates according to the different VOCs produced. "Fungal isolate" (including the PDA control) was the response factor, and a subset of all VOCs produced in significant amounts by the isolates (Table 2) was the predictor [2-non-anone; 1-octen-3ol; 3,5,5-trimethyl-2-hexene; germacrene D; $\alpha$-patcholene; 2-(1cyclopent-1-enyl-methylethyl)cyclopentanone; acoradiene; and mellein] to classify the isolates into their respective isolate groups. We omitted alpha-cedrene and dihydro-beta-iodine in the classification as these were strongly correlated with the other predictors. The fungal isolates were grouped into their respective disease groups (control, FHB, and SNB). The disease group FHB contained all $F$. graminearum, F. avenaceum, F. culmorum, and $F$. poae isolates, while the $\mathrm{SNB}$ group contained all $P$. nodorum isolates. Only octanal; 1-octen-3-ol; 3,5,5-trimethyl2-hexene; germacrene D; $\alpha$-patcholene; 2-(1-cyclopent-1-enylmethylethyl); and mellein were used as predictors for "disease" as we again omitted those VOCs that correlated strongly with other 
TABLE 2 | Volatile organic compounds in headspace collections from fungal isolates on potato dextrose agar (PDA).

\begin{tabular}{|c|c|c|c|c|c|c|c|c|c|c|c|c|c|c|}
\hline \multirow{4}{*}{$\begin{array}{l}\text { DB-WAX } \\
\text { RT }\end{array}$} & \multirow[t]{4}{*}{ RI } & \multirow{4}{*}{$\begin{array}{l}\text { Compound } \\
\text { (Identified by } \\
\text { AMDIS) }\end{array}$} & \multirow[t]{4}{*}{ CAS Nr } & \multirow[t]{4}{*}{$P$ value } & \multicolumn{10}{|c|}{ Amount of compounds trapped in $24 \mathrm{~h}$ are presented as microgram heptyl acetate equivalents } \\
\hline & & & & & \multicolumn{6}{|c|}{ Fusarium head blight ${ }^{c}$} & \multirow{2}{*}{\multicolumn{4}{|c|}{$\begin{array}{l}\text { Septoria nodorum blotch } \\
\text { Parastagonospora nodorum }\end{array}$}} \\
\hline & & & & & \multicolumn{3}{|c|}{ Fusarium graminearum } & \multirow{2}{*}{$\begin{array}{c}\begin{array}{c}\text { Fusarium } \\
\text { avenaceum }\end{array} \\
\begin{array}{l}203587 \\
(n=4)^{\mathrm{b}}\end{array}\end{array}$} & \multirow{2}{*}{$\begin{array}{c}\begin{array}{c}\text { Fusarium } \\
\text { culmorum }\end{array} \\
\begin{array}{l}202588 \\
(n=4)^{\mathrm{b}}\end{array}\end{array}$} & \multirow{2}{*}{$\begin{array}{c}\begin{array}{c}\text { Fusarium } \\
\text { poae }\end{array} \\
\begin{array}{c}202589 \\
(n=4)^{\mathrm{b}}\end{array}\end{array}$} & & & & \\
\hline & & & & & $\begin{array}{l}201569 \\
(n=3)^{b}\end{array}$ & $\begin{array}{c}\text { KISH } \\
140 / 80 \\
(n=3)^{b}\end{array}$ & $\begin{array}{l}\text { KISH } \\
61 / 07 \\
(n=3)^{b}\end{array}$ & & & & $\begin{array}{l}201254 \\
(n=4)^{b}\end{array}$ & $\begin{array}{l}201252 \\
(n=5)^{b}\end{array}$ & $\begin{array}{l}201253 \\
(n=5)^{b}\end{array}$ & $\begin{array}{l}202586 \\
(n=5)^{b}\end{array}$ \\
\hline 9.05 & 1,285 & Octanal ${ }^{\mathrm{a}}$ & 124-13-0 & 0.010 & 0 & 0 & 0 & 0.005 & 0 & 0.09 & 0 & 0 & 0 & 0 \\
\hline 11.09 & 1,390 & 2-Non-anone & $124-19-6$ & 0.042 & 0.088 & 0.052 & 0.052 & 0.05 & 0.056 & 0.05 & 0.222 & 0.066 & 0.043 & 0.064 \\
\hline 12.22 & 1,451 & 1-Octen-3-ola & $3391-86-4$ & $<0.001$ & 0.06 & 0.425 & 0.303 & 0.036 & 0.214 & 0.029 & 0.888 & 0.616 & 0.944 & 2.205 \\
\hline 12.84 & 1,482 & $\begin{array}{l}\text { 3,5,5-Trimethyl-2- } \\
\text { hexene }\end{array}$ & 26456-76-8 & $<0.001$ & 0.108 & 0.094 & 0.042 & 0.004 & 0.013 & 0.024 & 0.265 & 0.01 & 0.338 & 0.237 \\
\hline 13.58 & 1,523 & Sativene $^{a}$ & $3650-28-0$ & 0 & 0 & 0 & 0 & 0 & 0 & 0 & 0 & 0 & 0 & 0 \\
\hline 14.17 & 1,559 & 1-Octanol ${ }^{\mathrm{a}}$ & $111-87-5$ & $<0.001$ & 0 & 0 & 0 & 0 & 0 & 0.014 & 0 & 0 & 0 & 0 \\
\hline 14.26 & 1,564 & Alpha-cedrene & $469-61-4$ & 0.002 & 0 & 0 & & 0 & 0 & 0.033 & 0.057 & 0 & 0 & 0.054 \\
\hline 16.64 & 1,702 & Germacrene $D^{a}$ & $23986-74-5$ & $<0.001$ & 0.026 & 0.042 & 0 & 0 & 0.02 & 0.475 & 0.162 & 0 & 0 & 0.429 \\
\hline 17.44 & 1,748 & $\alpha$-Patcholene & $560-32-7$ & $<0.001$ & 0.03 & 0.03 & 0.047 & 0 & 0 & 0.357 & 0.166 & 0.011 & 0 & 0.288 \\
\hline 17.56 & 1,757 & $\begin{array}{l}\text { 2-(1-Cyclopent-1- } \\
\text { enyl-methylethyl) } \\
\text { cyclopentanone }\end{array}$ & NA (NIST\# 62949) & $<0.001$ & 0.115 & 0.118 & 0.359 & 0 & 0 & 1.219 & 0 & 0 & 0 & 0.046 \\
\hline 20.93 & 2,020 & 2-Pentadecanone & $2345-28-0$ & 0 & 0 & 0 & 0 & 0 & 0 & 0 & 0 & 0 & 0 & 0 \\
\hline 21.35 & 2,070 & Acoradiene & 24048-44-0 & $<0.001$ & 0 & 0 & 0 & 0 & 0 & 0.273 & 0 & 0 & 0 & 0 \\
\hline 21.50 & 2,088 & $\begin{array}{l}\text { Dihydro-beta- } \\
\text { ionone }\end{array}$ & $17283-81-7$ & $<0.001$ & 0 & 0 & 0 & 0 & 0 & 0.526 & 0 & 0 & 0 & 0 \\
\hline 22.42 & 2,232 & 2-Heptadecanone & $2922-51-2$ & 0 & 0 & 0 & 0 & 0 & 0 & 0 & 0 & 0 & 0 & 0 \\
\hline 23.75 & 2,507 & Mellein ${ }^{a}$ & $17397-85-2$ & $<0.001$ & 0 & 0 & 0 & 0 & 0 & 0 & 2.754 & 24.858 & 2.732 & 23.544 \\
\hline
\end{tabular}

Median is given in the table for the different compounds. If the P value was less than 0.05 , at least one median was different from the rest. Gray cells indicate which median of which compounds was above zero. ${ }^{a}$ Compounds in bold were verified by comparison with synthetic standards.

${ }^{b}$ Number of replicates is given in parenthesis after isolate number.

${ }^{c}$ Amount of compounds trapped in $24 \mathrm{~h}$ is presented as microgram heptyl acetate equivalents from different isolates grown on PDA in four 5-cm Petri dishes. Volatile organic compounds were collected from 6- to 10-day-old cultures. Due to non-normality, the use of the non-parametric Kruskal-Wallis test was warranted. P values were adjusted for ties. 
predictors. Cross validation was used to test the robustness of the classification system.

\section{Determining Which Volatile Organic Compounds Were Produced by Two Different Wheat Varieties Inoculated With Different Pathogens}

The median amounts for each of these selected VOCs for all replicates were calculated for each pathogen-variety-time after inoculation combination. Each experiment (pathogen-varietytime after inoculation combination) was repeated three-four times, except for the collection of VOCs from 'Bjarne' inoculated with PM at $14 \mathrm{dpi}$. The data was not normally distributed due to many zeros in the dataset. Transformation of the data did not improve normality. Therefore, we used a non-parametric Kruskal-Wallis test to test if the median amounts of each collected compound differed significantly between the treatments (FHB, SNB, control, and PM), separately for one wheat variety ('Bjarne' or 'Zebra') at each collection time (7, 14, and $21 \mathrm{dpi}$ ). This gave a total of 225 tests $\{[2$ varieties $\times 2$ diseases $(\mathrm{FHB}$ and $\mathrm{SNB}) \times 3$ collection times $\times 15$ compounds $]+[1$ variety $\times 1$ disease $(\mathrm{PM}) \times 3$ collection times $\times 15$ compounds]\}. $P$-values were adjusted for ties, and the significance level was set to 0.05 (Table 3).

\section{The Classification of Fungal Diseases Into Their Respective Disease Group}

We used a discriminant analysis based on a linear model to group the diseases on both varieties and the control 7 , 14 , and 21 dpi. We only considered those compounds in the discriminant analysis that were produced in significant amounts as predictors for each variety separately (Table 3 ). We found that 2-(1-cyclopen-1-enyl-methylethyl)cyclopentanone, dihydro-beta-iodine, $\alpha$-patcholene, and dihydro-beta-ionine were highly correlated with other VOCs at 14 and 21 dpi in the 'Zebra' samples, so we omitted these compounds in the discriminant analysis and only used 3,5,5-trimethyl-2-hexene; sativene; germacrene $\mathrm{D}$; and 2-heptadecanone to classify the diseases 7 and 14 dpi. Germacrene D was highly correlated with the other predictors at $21 \mathrm{dpi}$, and mellein was added to the predictors instead. For classification of diseases at $7 \mathrm{dpi}$ on 'Bjarne,' we used 1-octen-3-ol; 3,5,5-trimethyl-2-hexene; 1octanol; sativene; alpha-cedrene; germacrene D; 2-(1-cyclopent1-enyl-methylethyl)cyclopentanone; and 2-heptadecanone as the predictors. After $14 \mathrm{dpi}$, we classified the diseases on 'Bjarne' into their respective groups using 1-octen-3-ol; 3,5,5trimethyl-2-hexene; 1-octanol; sativene; alpha-cedrene; and 2heptadecanone. The classification of diseases on 'Bjarne' into their respective groups $21 \mathrm{dpi}$ was based on only 1-octen-3-ol; 3,5,5-trimethyl-2-hexene; alpha-cedrene; germacrene D; mellein; and $\alpha$-patcholene.

We then combined the VOC data for all sampling times for 'Zebra' (predictors included 3,5,5-trimethyl-2-hexene; sativene; germacrene D; mellein; and 2-heptadecanone) and did the same for 'Bjarne' [predictors included 1-octen-3ol; 3,5,5-trimethyl-2-hexene; alpha-cedrene; germacrene D; 2-(1-cyclopent-1-enyl-methylethethyl)cyclopentanone; 2pentadecanone; 2-heptadecanone; mullein; and 1-octanol] to see if the VOC blend-based classification was correctly putting the diseases into their respective groups independent of how long after infection the VOCs were collected from the two different varieties. Finally, we combined the data from all sample times and both varieties to see if the classification would work independent of the variety we used [predictors included 1-octen-3-ol; 3,5,5trimethyl-2-hexene; sativene; germacrene $D ; \alpha$-patcholene; 2-(1-cyclopent-1-enyl-methylethethyl)cyclopentanone; mellein; and 2-heptadecanone]. We used cross validation to test the robustness of the classification model.

\section{Correlation of Volatile Organic Compounds With Disease Index Across Wheat Varieties}

We regressed the DI of SNB, FHB, and PM with VOCs produced in significant amounts after inoculation from each and then from both wheat varieties over time. Based on the Table 3, we chose to regress mellein and heptadecanone against the DI of SNB as they were consistently produced by 'Zebra' and/or 'Bjarne' in significant amounts. For FHB, we chose germacrene $\mathrm{D}$ and sativene as they were the VOCs produced by 'Zebra' and/or 'Bjarne' at all collection times. Both 1-octen3-ol and 3,5,5-trimethyl-2-hexene were produced in significant amounts by 'Bjarne' inoculated with PM at most sampling times, and both compounds were regressed against the DI of PM. We compared the regression models for each disease-VOC relationship between the two different varieties using "variety" as a categorical predictor and "DI $\times$ variety" as the interaction term.

\section{RESULTS}

\section{Fungal Growth and Disease Severity in Plants}

The average colony area grown on four Petri dishes for each isolate at the time of VOC collection is given in Table 1. Isolates of F. culmorum, F. avenaceum, and F. poae and isolates KISH140/80 and KISH61/07 of F. graminearum covered the entire area of each Petri dish $\left(19.63 \mathrm{~cm}^{2}\right)$, amounting to $78.54 \mathrm{~cm}^{2}$ for four Petri dishes at each collection time, while F. graminearum isolate 201569 covered on average $44.00 \mathrm{~cm}^{2}$, and the four $P$. nodorum isolates covered an area from 25.73 to $43.98 \mathrm{~cm}^{2}$ per collection time (Table 1).

The average disease severity, fresh biomass, and the DI on wheat variety 'Bjarne' and 'Zebra' at the time of VOC collection and 7, 14, and 21 dpi with FHB, SNB, and/or PM are given in Table 4. The average FHB severity increased from 30 to 55\% and from 29 to $55 \%$ between day 7 and day 21 on 'Zebra' and 'Bjarne', respectively. The average SNB severity on 'Zebra' and 'Bjarne' increased from 1.6 to $14 \%$ between 7 and 21 days and from 4.3 to $32 \%$, during the same time, respectively. The average PM severity on 'Bjarne' almost doubled from $48 \%$ at 7 dpi to $84 \%$ at 21 dpi. There was no disease development visible on the control plants. On 'Zebra', the fresh weight from the FHB experiments varied between 48.25 and $59.3 \mathrm{~g}$ and from the SNB experiments between 46.5 and $53.26 \mathrm{~g}$. The fresh weight of 'Bjarne' plants used for each of the FHB experiments varied between 38.66 and $44.53 \mathrm{~g}$, between 35.97 and $56.49 \mathrm{~g}$ for the 
TABLE 3 | Volatile organic compounds in headspace collections from potted wheat plants ('Zebra' and 'Bjarne') 7 (A), 14 (B), and 21 (C) days after inoculation with Fusarium graminearum or Parastagonospora nodorum. Mean release rates of compounds trapped over $24 \mathrm{~h}$ are presented as heptyl equivalents per pot. The amounts of the different compounds are given in medians. If the $P$ value was less than 0.05 , one treatment median per variety was significantly different from the rest (Kruskal-Wallis test, gray cells). $P$ values were adjusted for ties. Only compounds that were produced in significantly higher amounts than from the control are listed in the table.

(A)

(20)

'Zebra'

'Bjarne'

\begin{tabular}{|c|c|c|c|c|c|c|c|c|c|c|}
\hline RT & $\mathbf{R I}$ & $\begin{array}{l}\text { Compound } \\
\text { (identified by AMDS) }\end{array}$ & Cas $\mathrm{Nr}$ & $P$ value & $\begin{array}{l}\text { P. nodorum } \\
(n=4)^{b}\end{array}$ & $\begin{array}{l}\text { F. graminearum } \\
\qquad(n=3)^{b}\end{array}$ & $P$ value & $\begin{array}{l}P . \text { nodorum } \\
(n=4)^{b}\end{array}$ & $\begin{array}{l}\text { F. graminearum } \\
\qquad(n=3)^{b}\end{array}$ & $\begin{array}{l}\text { B. graminis } \\
(n=3)^{\mathrm{b}}\end{array}$ \\
\hline 12.84 & 1,482 & $\begin{array}{l}\text { 3,5,5-Trimethyl-2- } \\
\text { hexene }\end{array}$ & $26456-76-8$ & 0.14 & 0.006 & 0.016 & 0.035 & 0.006 & 0.013 & 0.032 \\
\hline 13.58 & 1,523 & Sativene $^{a}$ & $3650-28-0$ & 0.02 & 0 & 0.019 & 0.01 & 0 & 0.021 & 0 \\
\hline 14.26 & 1,564 & Alpha-cedrene & $469-61-4$ & 0.17 & 0.001 & 0.012 & 0.026 & 0.004 & 0.012 & 0 \\
\hline 16.64 & 1,702 & Germacrene $\mathbf{D}^{\mathrm{a}}$ & $23986-74-5$ & 0.012 & 0 & 0.035 & 0.002 & 0 & 0.043 & 0 \\
\hline \multirow[t]{2}{*}{17.56} & 1,757 & $\begin{array}{l}\text { 2-(1-Cyclopent-1-enyl- } \\
\text { methylethyl) } \\
\text { cyclopentanone }\end{array}$ & NA & 0.005 & 0 & 0.256 & 0.002 & 0 & 0.253 & 0 \\
\hline & & & (NIST\# 62949) & & & & & & & \\
\hline 22.42 & 2,232 & 2-Heptadecanone & $2922-51-2$ & 0.039 & 0.045 & 0 & 0.002 & 0.021 & 0 & 0 \\
\hline
\end{tabular}

(B)

\begin{tabular}{|c|c|c|}
\hline 12.22 & 1,451 & 1-Octen-3-ola \\
\hline 12.84 & 1,482 & $\begin{array}{l}\text { 3.5.5-Trimethyl-2- } \\
\text { hexene }\end{array}$ \\
\hline 13.58 & 1,523 & Sativene $^{a}$ \\
\hline 16.64 & 1,702 & Germacrene $\mathbf{D}^{\mathrm{a}}$ \\
\hline 17.44 & 1,748 & $\alpha-\Pi \alpha \tau \chi \eta \circ \lambda \varepsilon \nu \varepsilon$ \\
\hline 17.56 & 1,757 & $\begin{array}{l}\text { 2-(1-Cyclopent-1-enyl- } \\
\text { methylethyl) } \\
\text { cyclopentanone }\end{array}$ \\
\hline 21.35 & 2,070 & Acoradiene \\
\hline 21.5 & 2,088 & Dihydro-beta-ionone \\
\hline 22.42 & 2,232 & 2-Heptadecanone \\
\hline 23.75 & 2,507 & Mellein ${ }^{a}$ \\
\hline
\end{tabular}

\begin{tabular}{|c|c|}
\hline $3391-86-4$ & 0.06 \\
\hline $26456-76-8$ & 0.018 \\
\hline $3650-28-0$ & 0.005 \\
\hline $23986-74-5$ & 0.013 \\
\hline $560-32-7$ & 0.005 \\
\hline NA & 0.005 \\
\hline
\end{tabular}

$\begin{array}{ccc}0.029 & 0.117 & 0.036 \\ 0.007 & 0.038 & 0.018 \\ 0 & 0.007 & 0.06 \\ 0 & 0.069 & 0.005 \\ 0 & 0.011 & 0.06 \\ 0 & 0.149 & 0.014 \\ & & \end{array}$

$\begin{array}{cc}0.036 & 0.022 \\ 0.018 & 0.006 \\ 0.06 & 0 \\ 0.005 & 0 \\ 0.06 & 0 \\ 0.014 & 0\end{array}$

(NIST\# 62949)

24048-44-0 0.005

17283-81-7 0.005

2922-51-2 $\quad 0.084 \quad 0.051$

$\begin{array}{lll}17397-85-2 & 0.033 & 0.003\end{array}$

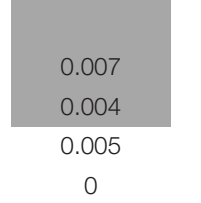

0.06
0.06
0.016
0.038

0
0
0.039
0.006

$\begin{array}{cc}0.006 & 0 \\ 0.003 & 0 \\ 0 & 0 \\ 0 & 0\end{array}$

(C)

\begin{tabular}{|c|c|c|}
\hline 12.22 & 1,451 & 1-Octen-3-ol \\
\hline 12.84 & 1,482 & $\begin{array}{l}\text { 3,5,5-Trimethyl-2- } \\
\text { hexene }\end{array}$ \\
\hline 13.58 & 1,523 & Sativene $^{a}$ \\
\hline 14.26 & 1,564 & Alpha-cedrene \\
\hline 16.64 & 1,702 & Germacrene $\mathbf{D}^{\mathrm{a}}$ \\
\hline 17.44 & 1,748 & $\alpha-\Pi \alpha \tau \chi \eta \circ \lambda \varepsilon v \varepsilon \varepsilon$ \\
\hline 17.56 & 1,757 & $\begin{array}{l}\text { 2-(1-Cyclopent-1-enyl- } \\
\text { methylethyl) } \\
\text { cyclopentanone }\end{array}$ \\
\hline
\end{tabular}

20.93 2,020 2-Pentadecanone

21.35 2,070 Acoradiene

$21.52,088$ Dihydro-beta-ionone

22.42 2,232 2-Heptadecanone

23.75 2,507 Mellein ${ }^{a}$

$\begin{array}{ccc}3391-86-4 & 0.068 & 0.084 \\ 26456-76-8 & 0.036 & 0.009 \\ 3650-28-0 & 0.005 & 0 \\ 469-61-4 & 0.049 & 0.006 \\ 23986-74-5 & 0.005 & 0 \\ 560-32-7 & 0.005 & 0 \\ \text { NA } & 0.005 & 0 \\ & & \end{array}$

\begin{tabular}{l}
0.134 \\
0.029 \\
0.014 \\
0.013 \\
0.073 \\
0.018 \\
0.238 \\
\hline
\end{tabular}

0.018
0.013
0.046
0.022
0.016
0.148
0.065

0.047
0.01
0
0.005
0
0
0

0.101

0.026

0.006
0.014
0.045
0.009
0.11

0.781
0.352
0
0
0
0
0.014

(NIST\# 62949)

\begin{tabular}{cccccccc}
$2345-28-0$ & 0.113 & 0.57 & 0.103 & 0.024 & 0.31 & 0.734 & 0 \\
$24048-44-0$ & 0.005 & 0 & 0.012 & 0.046 & 0 & 0.008 & 0 \\
$17283-81-7$ & 0.005 & 0 & 0.004 & 0.046 & 0 & 0.003 & 0 \\
$2922-51-2$ & 0.116 & 0.014 & 0 & 0.003 & 0.033 & 0 \\
$17397-85-2$ & 0.033 & 0.003 & 0 & 0.003 & 0.027 & 0 \\
\hline
\end{tabular}

aCompounds in bold were verified by comparison with synthetic standards.

${ }^{b}$ Number of replicates are given in parenthesis after the name of the disease. 
SNB experiments, and from 4.64 to $5.71 \mathrm{~g}$ for seedlings in the PM experiments. Accordingly, the DI across disease, variety, and day after inoculation combinations varied considerably between 1,068 and 3.434 for FHB and between 99 and 702 for SNB on 'Zebra' between 7 and 21 dpi. On 'Bjarne,' the DI for FHB during the same time interval varied between 1,394 and 2,237, for SNB between 134 and 1,830, and for PM between 246 and 521 (Table 4). There was no significant increase in the average disease severity, fresh biomass, or DI for FHB and SNB on 'Zebra' and FHB on 'Bjarne.' However, these parameters increased significantly with time after inoculation for SNB on 'Bjarne.'

\section{Volatile Organic Compounds From Fungal Isolates in Pure Culture}

MetAlign grouping selected the following 15 compounds to be significant in distinguishing between fungal isolates on PDA and control (PDA only): octanal; 2-non-anone; 1octen-3-ol; 3,5,5-trimethyl-2-hexene; 1-octanol; alpha-cedrene; sativene; germancrene $\mathrm{D} ; \alpha$-patcholene; 2-(1-cyclopent-1-enylmethylethyl)cyclopentanone; acoradiene; dihydro-beta-ionone; 2-pentadecanone; 2-heptadecanone; and mellein. The retention time, retention index, and CAS number [a unique number assigned by the Chemical Abstracts Service (CAS)] of the 15 compounds are presented in Table 2. The compound 1octen-3-ol was sampled in significant amounts from all isolates tested on PDA, except from F. avenaceum, while 2-non-anone was sampled from all isolates except from $F$. graminearum KISH140/80 and KISH61/07. The compound 3,5,5-trimethyl-2hexene was sampled from all isolates except from $F$. avenaceum and F. culmorum (Table 2).

Most isolates emitted a unique blend of VOCs (Table 2). F. graminearum isolate 201569 emitted 2-non-anone; 1-octen3-ol; and 3,5,5-trimethyl-2-hexene. Both F. graminearum isolate $140 / 80$ and 61/07 emitted 1-octen-3-ol; 3,5,5-trimethyl-2hexene; and 2-(1-cyclopent-1-enyl-methylethyl)cyclopentanone, but isolate140/80 emitted germacrene D in addition to those compounds. F. avenaceum (isolate 203587) emitted only 2non-anone, and F. culmorum (isolate 202588) emitted 2non-anone and 1-octen-3-ol. F. poae (isolate 202589) emitted octanal; 2-non-anone; 1-octen-3-ol; 3,5,5-trimethyl-2-hexene; alpha-cedrene; germancrene D; $\alpha$-patcholene; 2-(1-cyclopent-1enyl-methylethyl)cyclopentanone; acoradiene; and dihydro-betaionone (Table 2).

All four isolates from the SNB pathogen $P$. nodorum produced 2-non-anone; 1-octen-3-ol; 3,5,5-trimethyl-2-hexene; and mellein. In addition, both P. nodorum isolate 201254 and isolate 202586 produced alpha-cedrene, germacrene $\mathrm{D}$, and $\alpha$ patcholene; isolate 201252 produced $\alpha$-patcholene; and isolate 201253 produced no other additional VOCs (Table 2).

Linear discriminant analysis based on a linear model with "isolate" as the response and all compounds produced in significant amounts included as predictors [octanal; 2-nonanone; 1-octen-3-ol; 3,5,5-trimethyl-2-hexene; germacrene D; $\alpha$ patcholene; 2-(1-cyclopent-1-enyl-methylethyl; acoradiene; and mellein as the predictors] showed an overall correct classification of $84.2 \%$. Cross validation showed that $68.4 \%$ of isolates were 
classified correctly based on these nine VOCs chosen for the analysis. F. graminearum isolate 201569 and isolate KISH140/80, F. poae, P. nodorum isolate 201254, and the PDA control were classified $100 \%$ correctly into their isolate group, while P. nodorum isolate 201253, F. avenaceum, and F. graminearum isolate KISH61/70 were classified 80,75 , and $67 \%$ correctly (Table 5). P. nodorum isolate 202586, P. nodorum isolate 201252, and F. culmorum were classified 60,50 , and $50 \%$ correctly into their fungal isolate group, respectively, based on the selected volatiles (Table 5). Cross validation showed a lower level of correct classification than during the initial analysis except for F. avenaceum and F. graminearum isolates KISH140/80 and KISH61/70, which was correctly classified in 75,100 , and $67 \%$ of the cases, respectively (Table 5). Correct classification with cross validation was $25 \%$ for F. culmorum, $67 \%$ for F. graminearum isolate $201569,75 \%$ for $F$. poae, and $88 \%$ for the PDA control plates (Table 5). P. nodorum isolates 202586, 201253, 201252, and 201254 were correctly classified in $60,40,25$, and $80 \%$ of all cases, respectively, after cross validation. Then, we combined the F. graminearum, F. avenaceum, F. culmorum, and F. poae isolates into one group of Fusarium head blight pathogens and all $P$. nodorum isolates into the SNB group. After this grouping of the isolates into their disease groups, we had an overall correct classification into disease groups in 48 out of 57 cases or $84.2 \%$, when we used "disease group" as the response and 1-octen-3ol; 3,5,5-trimethyl-2-hexene; germacrene D; $\alpha$-patcholene; 2-(1cyclopent-1-enyl-methylethyl); and mellein as the predictors. All 17 controls were placed correctly into the control group. Six of the FHB isolates were also grouped into the control group, while 15 were grouped correctly into the FHB disease group (71.4\%). Sixteen out of 19 SNB isolates were correctly placed into the SNB group, while two were placed into the FHB group and one into the control group ( $84.2 \%$ correct classification) (Table 6). Based on the selected compounds, cross validation showed a somewhat lower overall rate of correct classification, $77.2 \%$, with $61.9 \%$ correct classification of FHB isolates and $73.7 \%$ correct classification of SNB isolates (Table 6).

\section{Volatile Organic Compounds From Inoculated Plants Identity of Volatile Organic Compounds Produced}

MetAlign grouping selected the following 12 compounds to be significant in distinguishing between inoculated and control plants at 7, 14, and 21 dpi (Table 3). Each disease on 'Zebra' and 'Bjarne' had a distinct VOC profile. The production of some

TABLE 5 | summary of classification for fungal isolates in pure culture based on linear discriminant analysis with each fungal isolate as the response factor and octanal; 2-non-anone; 1-octen-3-ol; 3,5,5-trimethyl-2-hexene; germacrene D; $\alpha$-patcholene; 2-(1-cyclopent-1-enyl-methylethyl) cyclopentanone; acoradiene, and mellein as the predictors.

\begin{tabular}{|c|c|c|c|c|c|c|c|c|c|c|c|}
\hline \multirow[b]{2}{*}{ Put into group } & \multicolumn{11}{|c|}{ True group } \\
\hline & $\begin{array}{c}F . \\
\text { avenaceun }\end{array}$ & $\begin{array}{c}\text { F. } \\
\text { n culmorum }\end{array}$ & $\begin{array}{c}F \\
\text { graminearum } \\
(201569)\end{array}$ & $\begin{array}{c}F . \\
\text { graminearu } \\
\text { (KISH } \\
\text { 140/80) }\end{array}$ & $\begin{array}{c}F . \\
\text { raminearum } \\
\text { (KISH } \\
61 / 70)\end{array}$ & F. poae & PDA & $\begin{array}{l}\text { P. nodorum } \\
\text { (202586) }\end{array}$ & $\begin{array}{c}\text { P. nodorum } \\
\text { (201253) }\end{array}$ & $\begin{array}{c}\text { P. nodorum } \\
\text { (201252) }\end{array}$ & $\begin{array}{c}\text { P. nodorum } \\
\text { (201254) }\end{array}$ \\
\hline F. avenaceum & 3 & 2 & 0 & 0 & 0 & 0 & 0 & 0 & 0 & 0 & 0 \\
\hline F. culmorum & 1 & 2 & 0 & 0 & 0 & 0 & 0 & 1 & 1 & 0 & 0 \\
\hline $\begin{array}{l}\text { F. graminearum } \\
(201569)\end{array}$ & 0 & 0 & 3 & 0 & 0 & 0 & 0 & 0 & 0 & 2 & 0 \\
\hline $\begin{array}{l}\text { F. graminearum } \\
\text { (KISH61/80) }\end{array}$ & 0 & 0 & 0 & 3 & 1 & 0 & 0 & 0 & 0 & 0 & 0 \\
\hline $\begin{array}{l}\text { F. graminearum } \\
\text { (KISH61/70) }\end{array}$ & 0 & 0 & 0 & 0 & 2 & 0 & 0 & 0 & 0 & 0 & 0 \\
\hline F. poae & 0 & 0 & 0 & 0 & 0 & 4 & 0 & 0 & 0 & 0 & 0 \\
\hline$P D A$ & 0 & 0 & 0 & 0 & 0 & 0 & 17 & 1 & 0 & 0 & 0 \\
\hline $\begin{array}{l}\text { P. nodorum } \\
\text { (202586) }\end{array}$ & 0 & 0 & 0 & 0 & 0 & 0 & 0 & 3 & 0 & 0 & 0 \\
\hline $\begin{array}{l}\text { P. nodorum } \\
\text { (201253) }\end{array}$ & 0 & 0 & 0 & 0 & 0 & 0 & 0 & 0 & 4 & 0 & 0 \\
\hline $\begin{array}{l}\text { P. nodorum } \\
\text { (201252) }\end{array}$ & 0 & 0 & 0 & 0 & 0 & 0 & 0 & 0 & 0 & 2 & 0 \\
\hline $\begin{array}{l}\text { P. nodorum } \\
\text { (201254) }\end{array}$ & 0 & 0 & 0 & 0 & 0 & 0 & 0 & 0 & 0 & 0 & 5 \\
\hline Total N & 4 & 4 & 3 & 3 & 3 & 4 & 17 & 5 & 5 & 4 & 5 \\
\hline Correct $N$ & 3 & 2 & 3 & 3 & 2 & 4 & 17 & 3 & 4 & 2 & 5 \\
\hline $\begin{array}{l}\text { Correctly classified } \\
(\%)\end{array}$ & 75 & 50 & 100 & 100 & 67 & 100 & 100 & 60 & 80 & 50 & 100 \\
\hline $\begin{array}{l}\text { Correctly classified } \\
\text { after cross } \\
\text { validation }\end{array}$ & 75 & 25 & 67 & 100 & 67 & 75 & 88 & 60 & 40 & 25 & 80 \\
\hline
\end{tabular}


TABLE 6 | Summary of classification for fungal isolates grouped into diseases based on linear discriminant analysis with disease group as a response and 1-octen-3-ol; 3,5,5-trimethyl-2-hexene; germacrene D; $\alpha$-patcholene; 2-(1-cyclopent-1-enyl-methylethyl)cyclopentanone; and mellein as the predictors.

Put into group

\begin{tabular}{lccc}
\cline { 2 - 4 } & Control & $\begin{array}{c}\text { Fusarium } \\
\text { head blight }\end{array}$ & $\begin{array}{c}\text { Septoria nodorum } \\
\text { blotch }\end{array}$ \\
\hline Control & 17 & 6 & 1 \\
Fusarium head blight & 0 & 15 & 2 \\
Septoria nodorum blotch & 0 & 0 & 16 \\
Total $N$ & 17 & 21 & 19 \\
Correct $N$ & 17 & 15 & 16 \\
Correctly classified (\%) & 100 & 71.4 & 84.2 \\
Correct after cross validation (\%) & 100 & 61.9 & 73.7
\end{tabular}

compounds was only observed at some, but not all, collection times. 'Zebra' and 'Bjarne' both emitted sativene, germacrene D, and 2-(1-cyclopent-1-enyl-methylethyl)cyclopentanone 7 days after inoculation with $F$. graminearum. However, while 'Zebra' produced 3,5,5-trimethyl-2-hexene, 'Bjarne' produced alphacedrene in addition to those compounds 7 days after inoculation (Table 3A). Both cultivars produced 2-heptadecanone 7 days after inoculation with $P$. nodorum (Table 3A). Seven days after inoculating 'Bjarne' with the powdery mildew pathogen B. graminis, the plants emitted 3,5,5-trimethyl-2-hexene.

After 14 days of inoculation with F. graminearum, 'Zebra' and 'Bjarne' both still produced germacrene D and 2-(1-cyclopent1-enyl-methylethyl)cyclopentanone, while only 'Zebra' produced sativene. In addition, 'Zebra' also produced 3,5,5-trimethyl-2hexene, $\alpha$-patcholene, acoradiene, and dihydro-beta-ionone after inoculation with F. graminearum (Table 3B). Fourteen days after inoculation with $P$. nodorum, both varieties produced mellein, but 'Bjarne' also produced 2-heptadecanone in addition to that (Table 3B). 'Bjarne' produced 1-octen-3-ol and 3,5,5-trimethyl2-hexene 14 dpi with B. graminis.
Twenty-one days after inoculating 'Zebra' and 'Bjarne' with F. graminearum, both varieties produced again sativene and germacrene D (Table 3C). In addition, 'Zebra' produced $\alpha$ patcholene, 2-(1-cyclopent-1-enyl-methylethyl)cyclopentanone, acoradiene, and dihydro-beta-ionone. 'Bjarne' plants produced alpha-cedrene and 2-pentadecanone in addition to sativene and germacrene at $21 \mathrm{dpi}$ with $F$. graminearum (Table 3C). Twenty-one days after inoculation with $P$. nodorum, 'Zebra' and 'Bjarne' both produced mellein, while 'Bjarne' also produced 2-heptadecanone in significant amounts (Table 3C). After 21 days, plants inoculated with B. graminis produced 1-octen-3-ol and 3,5,5-trimethyl-2-hexene in significant amounts (Table 3C).

\section{Classification of Fungal Diseases Into Their Respective Disease Group}

The discriminant analysis of the two diseases FHB and SNB on 'Zebra' with "disease" ("control," "FHB," and "SNB") as response factor and several selected volatiles as predictors correctly classified the diseases in 79,86 , and $86 \%$ for 7,14 , and $21 \mathrm{dpi}$, respectively. All "control" cases 7 and 14 dpi were correctly classified, while one of the seven control cases was classified as SNB at 21 dpi (Table 7). Fusarium head blight on 'Zebra' was $100 \%$ correctly classified at 14 and $21 \mathrm{dpi}$ and $67 \%$ at $7 \mathrm{dpi}$ (Table 7). SNB was correctly classified in $50 \%$ of the cases at 7 and $14 \mathrm{dpi}$ and in $75 \%$ at $21 \mathrm{dpi}$ (Table 7). Cross validation revealed a lower rate of correct classification with only $86 \%$ correct classifications of the control cases at all collection times. At 7, 14, and $21 \mathrm{dpi} 33,67$, and $67 \%$ of FHB cases were correctly classified, respectively, while 50,55, and 50\% of SNB cases were correctly classified at those time points using cross validation (Table 7). The discriminant analysis of the three different diseases on 'Bjarne,' with "disease" ("control," "FHB," "PM," and "SNB”) as response factor and different volatiles as predictors, classified $85 \%, 94 \%$, and $95 \%$ of the cases in their correct groups after 7 , 14 , and 21 dpi, respectively. From the 9 to 10 control cases at 7 , 14 , and $21 \mathrm{dpi}$, all were correctly placed in their control group.

TABLE 7 | Summary of classification of fungal diseases on 'Zebra' with "disease" [control, Fusarium head blight (FHB), and Septoria nodorum blotch (SNB)] as the response and different VOCs as predictors.

\begin{tabular}{|c|c|c|c|c|c|c|c|c|c|}
\hline \multirow[b]{3}{*}{ Put into group } & \multicolumn{9}{|c|}{ True group } \\
\hline & \multicolumn{3}{|c|}{7 days after inoculation ${ }^{a}$} & \multicolumn{3}{|c|}{14 days after inoculation } & \multicolumn{3}{|c|}{21 days after inoculation ${ }^{b}$} \\
\hline & Control & FHB & SNB & Control & FHB & SNB & Control & FHB & SNB \\
\hline Control & 7 & 1 & 2 & 7 & 0 & 2 & 6 & 0 & 1 \\
\hline $\mathrm{FHB}$ & 0 & 2 & 0 & 0 & 3 & 0 & 0 & 3 & 0 \\
\hline SNB & 0 & 0 & 2 & 0 & 0 & 2 & 1 & 0 & 3 \\
\hline Total N & 7 & 3 & 4 & 7 & 3 & 4 & 7 & 3 & 4 \\
\hline Correct $N$ & 7 & 2 & 2 & 7 & 3 & 2 & 6 & 3 & 3 \\
\hline Correctly classified (\%) & 100 & 67 & 50 & 100 & 100 & 50 & 86 & 100 & 75 \\
\hline Correctly classified (\%) after cross validation & 86 & 33 & 50 & 86 & 67 & 55 & 86 & 67 & 50 \\
\hline
\end{tabular}

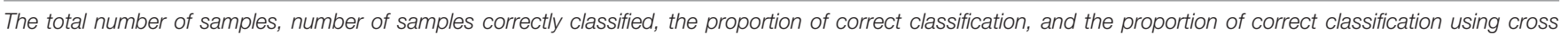
validation are given in the table.

aAfter 7 and 14 days of inoculation, 3,5,5-trimethyl-2-hexene; sativene; germacrene D; and 2-heptadecanone were included as predictors.

${ }^{b}$ Germacrene $D$ was strongly correlated with other predictors at $21 \mathrm{dpi}$ and was therefore not included as predictor for that time point. 
TABLE 8 | Summary of classification of fungal diseases on 'Bjarne' with "disease" [control, Fusarium head blight (FHB), Septoria nodorum blotch (SNB), and powdery mildew (PM)] as the response and different VOCs as the predictors.

\begin{tabular}{|c|c|c|c|c|c|c|c|c|c|c|c|c|}
\hline \multirow[b]{3}{*}{ Put into group } & \multicolumn{12}{|c|}{ True group } \\
\hline & \multicolumn{4}{|c|}{7 days after inoculation } & \multicolumn{4}{|c|}{14 days after inoculation ${ }^{b}$} & \multicolumn{4}{|c|}{21 days after inoculation ${ }^{c}$} \\
\hline & Control & FHB & SNB & PM & Control & FHB & SNB & PM & Control & FHB & SNB & PM \\
\hline Control & 10 & 1 & 1 & 1 & 9 & 1 & 1 & 0 & 9 & 0 & 1 & 0 \\
\hline FHB & 0 & 2 & 0 & 0 & 0 & 2 & 0 & 0 & 0 & 3 & 0 & 0 \\
\hline SNB & 0 & 0 & 3 & 0 & 0 & 0 & 3 & 0 & 0 & 0 & 3 & 0 \\
\hline PM & 0 & 0 & 0 & 2 & 0 & 0 & 0 & 2 & 0 & 0 & 0 & 3 \\
\hline Total N & 10 & 3 & 4 & 3 & 9 & 3 & 4 & 2 & 9 & 3 & 4 & 3 \\
\hline Correct $N$ & 10 & 2 & 3 & 2 & 9 & 2 & 3 & 2 & 9 & 3 & 3 & 3 \\
\hline Correctly classified (\%) & 100 & 67 & 75 & 67 & 100 & 67 & 75 & 100 & 100 & 100 & 75 & 100 \\
\hline Correctly classified (\%) after cross validation & 90 & 67 & 25 & 67 & 100 & 67 & 75 & 100 & 89 & 33 & 50 & 67 \\
\hline
\end{tabular}

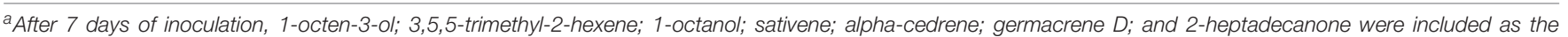
predictors for classification of VOCs.

${ }^{b}$ After 14 days of inoculation, the predictors included 1-octen-3-ol; 3,5,5-trimethyl-2-hexene; sativene; 2-heptadecanone; and mullein.

${ }^{c}$ After 21 days of inoculation, the predictors included 1-octen-3-ol; 3,5,5-trimethyl-2-hexene; alpha-cedrene; germacrene D; $\alpha$-patcholene; and mullein.

However, one of the FHB cases was also placed in the control group after 7 and 14 dpi (67\% correct) (Table 8). Twenty-one days after inoculation, all FHB cases on 'Bjarne' were correctly placed into the FHB group (Table 8). At all collection times, SNB on 'Bjarne' was correctly placed into the SNB group in $75 \%$ of the cases (Table 8). Powdery mildew cases were 100\% correctly classified after 14 and $21 \mathrm{dpi}$, while one PM case was placed in the control group after $7 \mathrm{dpi}$ (67\% correct, Table 8). Cross validation showed a lower percentage of correct classification for all diseases at 7, 14, and $21 \mathrm{dpi}$, except for FHB at 7 and $14 \mathrm{dpi}, \mathrm{SNB}$ at $14 \mathrm{dpi}$, and PM at 7 and 14 dpi (Table 8).

When we combined all collection times for 'Zebra' and grouped the diseases based on the discriminant analysis, with the relevant VOCs as predictors and "control," "FHB," and "SNB" as response, $74 \%$ of the diseases cases were correctly classified. Ninety-one percent of the control cases were correctly classified, while $78 \%$ of FHB and $42 \%$ of SNB cases were correctly classified (Table 9). Cross validation showed the same results (Table 9). When we then combined all collection times for 'Bjarne' and grouped the diseases based on the discriminant analysis using different VOCs as predictors and "control," "FHB," "SNB," and "PM" as responses, we classified $86 \%$ of all disease cases correctly. All of the control cases were correctly grouped into the control class $(100 \%)$, while $78 \%$ of $\mathrm{FHB}, 75 \%$ of SNB, and $63 \%$ of PM cases were correctly classified (Table 9). Cross validation returned the same results except for SNB, when only $67 \%$ instead of $75 \%$ of the disease cases were correctly grouped (Table 9).

Once we combined the data from both varieties and all collection times, using various VOCs as the predictors and "control," "FHB," "SNB," and "PM" as the responses, the discriminant analysis placed $80 \%$ of the disease cases correctly into their respective disease group. Ninety-six percent of control, $78 \%$ of $\mathrm{FHB}, 54 \%$ of $\mathrm{SNB}$, and $63 \%$ of PM cases were correctly grouped (Table 10). Cross validation returned similar results (Table 10). The classification of FHB and SNB based on VOCs produced by 'Zebra' (66.7 and 50\%, respectively) (Table 7) and 'Bjarne' (67 and 75\%, respectively) (Table 8) at 7 dpi was less
TABLE 9 | Summary of classification of fungal diseases on 'Zebra' and 'Bjarne,' when data from 7,14 , and 21 days after inoculation was combined.

\begin{tabular}{|c|c|c|c|c|c|c|c|}
\hline \multirow[b]{3}{*}{ Put into group } & \multicolumn{7}{|c|}{ True group } \\
\hline & \multicolumn{3}{|c|}{ Zebra $^{a}$} & \multicolumn{4}{|c|}{ Bjarne $^{b}$} \\
\hline & Control & FHB & SNB & Control & FHB & SNB & PM \\
\hline Control & 19 & 2 & 7 & 28 & 2 & 3 & 3 \\
\hline $\mathrm{FHB}$ & 0 & 7 & 0 & 0 & 7 & 0 & 0 \\
\hline SNB & 2 & 0 & 5 & 0 & 0 & 9 & 0 \\
\hline PM & - & - & & 0 & 0 & 0 & 5 \\
\hline Total $N$ & 21 & 9 & 12 & 28 & 9 & 12 & 8 \\
\hline Correct $N$ & 19 & 7 & 5 & 28 & 7 & 9 & 5 \\
\hline $\begin{array}{l}\text { Proportion of } \\
\text { correct N (\%) }\end{array}$ & 91 & 78 & 42 & 100 & 78 & 75 & 63 \\
\hline $\begin{array}{l}\text { Correctly classified } \\
\text { (\%) after cross } \\
\text { validation }\end{array}$ & 91 & 78 & 42 & 100 & 78 & 67 & 63 \\
\hline
\end{tabular}

Control, Fusarium head blight (FHB) and Septoria nodorum blotch (SNB) were the response factors, and different VOCs were the predictors for the classification of VOCs collected from 'Zebra.' Control, FHB, SNB, and powdery mildew (PM) were the response factors and different VOCs were the predictors for the classification of VOCs collected from 'Bjarne.'

a3,5,5-Trimethyl-2-hexene; sativene; germacrene D, mellein; and 2-heptadecanone were set as the predictors for classification of VOCs collected from 'Zebra.'

b1-Octen-3-ol; 3,5,5-trimethyl-2-hexene; alpha-cedrene; germacrene D; 2-(1cyclopent-1-enyl-methylethethyl)cyclopentanone; 2-heptadecanone; and mellein were the predictors for classification of VOCs collected from 'Bjarne.'

accurate than the grouping based on these VOCs produced at 21 dpi [100 and 75\% for 'Zebra' (Table 7) and 100 and 75\% for 'Bjarne' (Table 8)].

\section{The Relationship Between Volatile Organic Compound Emission and Disease Severity Across Wheat Varieties in vivo}

The regression analysis of emitted volatile compounds and their associated DI in both varieties showed generally a 
TABLE 10 | Summary of classification of fungal diseases on wheat (when data sets from 'Bjarne' and 'Zebra' 7, 14, and 21 days after inoculation were combined).

\begin{tabular}{lcccc}
\hline & \multicolumn{4}{c}{ True group } \\
\cline { 2 - 5 } & \multicolumn{4}{c}{ Zebra/Bjarne } \\
\cline { 2 - 5 } Put into group & Control (\%) & FHB & SNB & PM \\
\hline Control & 47 & 4 & 11 & 3 \\
FHB & 0 & 14 & 0 & 0 \\
PM & 0 & 0 & 0 & 5 \\
SNB & 2 & 0 & 13 & 0 \\
Total N & 49 & 18 & 24 & 8 \\
Correct N & 47 & 14 & 13 & 5 \\
Correctly classified (\%) & 96 & 78 & 54 & 63 \\
Correctly classified after cross validation (\%) & 94 & 72 & 50 & 63
\end{tabular}

"Disease" [including Fusarium head blight (FHB), Septoria nodorum blotch (SNB), and powdery mildew (PM)] was the response factor and 1-octen-3-ol; 3,5,5-trimethyl-2-hexene; sativene; germacrene D; $\alpha$-patcholene; 2-(1-cyclopent1-enyl-methylethethyl)cyclopentanone; mellein; and 2-heptadecanone were set as the predictors.

positive correlation. On 'Zebra', FHB DI and germacrene D were significantly correlated and FHB DI explained 44\% of the variation in germacrene $\mathrm{D}$ amounts (germacrene $\mathrm{D}_{\text {Zebra }}=0.0154+0.000014$ FHB DI, $\left.R^{2}=0.44, P=0.005\right)$, while FHB DI was not significantly correlated with sativene (sativene $_{\text {Zebra }}=0.0031+0.000004 \mathrm{FHB} \mathrm{DI} ; R^{2}=0.18, P=0.078$; Figure 2A). On 'Bjarne,' FHB DI was also significantly correlated with germacrene $\mathrm{D}$ and explained more than $85 \%$ of variation of this compound (germacrene $\mathrm{d}_{\text {Bjarne }}=0.0035+0.000016$ FHB DI, $R^{2}=0.86 \%, P<0.001$ ), while the production of sativene was also significantly correlated with FHB DI on 'Bjarne' (Figure 2B). However, FHB DI explained only 32\% of variation in sativene ( sativene $_{\text {Bjarne }}=0.0008+0.000005 \mathrm{FHB} \mathrm{D}, R^{2}=0.32$, $P=0.014$; Figure 2B). Both on 'Zebra' and 'Bjarne', the SNB DI was significantly correlated with mellein, while the SNB DI was only significantly correlated with 2-heptadecanone on 'Zebra' $(P=0.037)$ and not on 'Bjarne' $(P=0.057)$. The SNB DI explained $73 \%$ of variation in mellein production on both 'Zebra' and on 'Bjarne' (mellein Zebra $=-0.0016+0.000016$ SNB DI, $R^{2}=0.73 \%$, $P<0.001$; Figure 2C and mellein $_{\text {Bjarne }}=-0.0017+0.000013$ SNB DI, $R^{2}=0.73 \%, P<0.001$; Figure 2D). On 'Zebra', the SNB DI explained only $18 \%$ of variation in 2-heptadecanone (2heptadecanone $_{\text {Zebra }}=0.0208+0.000018$ SNB DI, $R^{2}=0.18 \%$, $P=0.037$; Figure 2C).

The analysis of variance of the regression analysis for the different VOCs and their respective DI showed that there was no significant difference between the VOC-DI relationships of the two varieties 'Zebra' and 'Bjarne.'

Once we combined the data for VOC production during the different times after inoculation for both varieties,
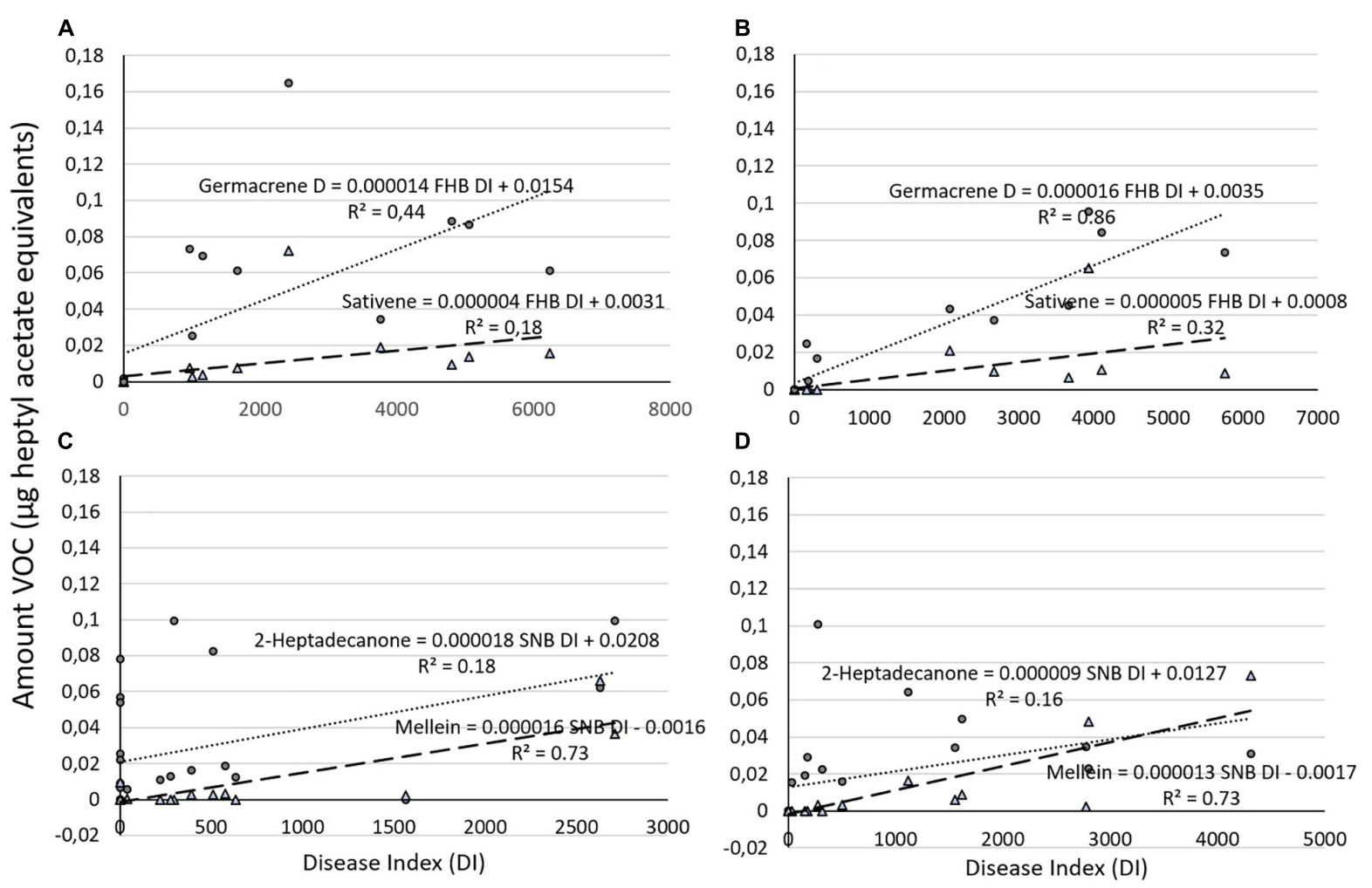

FIGURE 2 | Graphical representation of amount of (A) two different volatile organic compounds (VOCs) (germacrene and sativene) regressed against Fusarium head blight (FHB) DI on 'Zebra' and (B) on 'Bjarne' and (C) of two different VOCs (2-heptadecanone and mellein) against Septoria nodorum blotch (SNB) DI on 'Zebra' and on (D) 'Bjarne.' The regression equations and the $R^{2}$ values are placed next to the regression lines. 

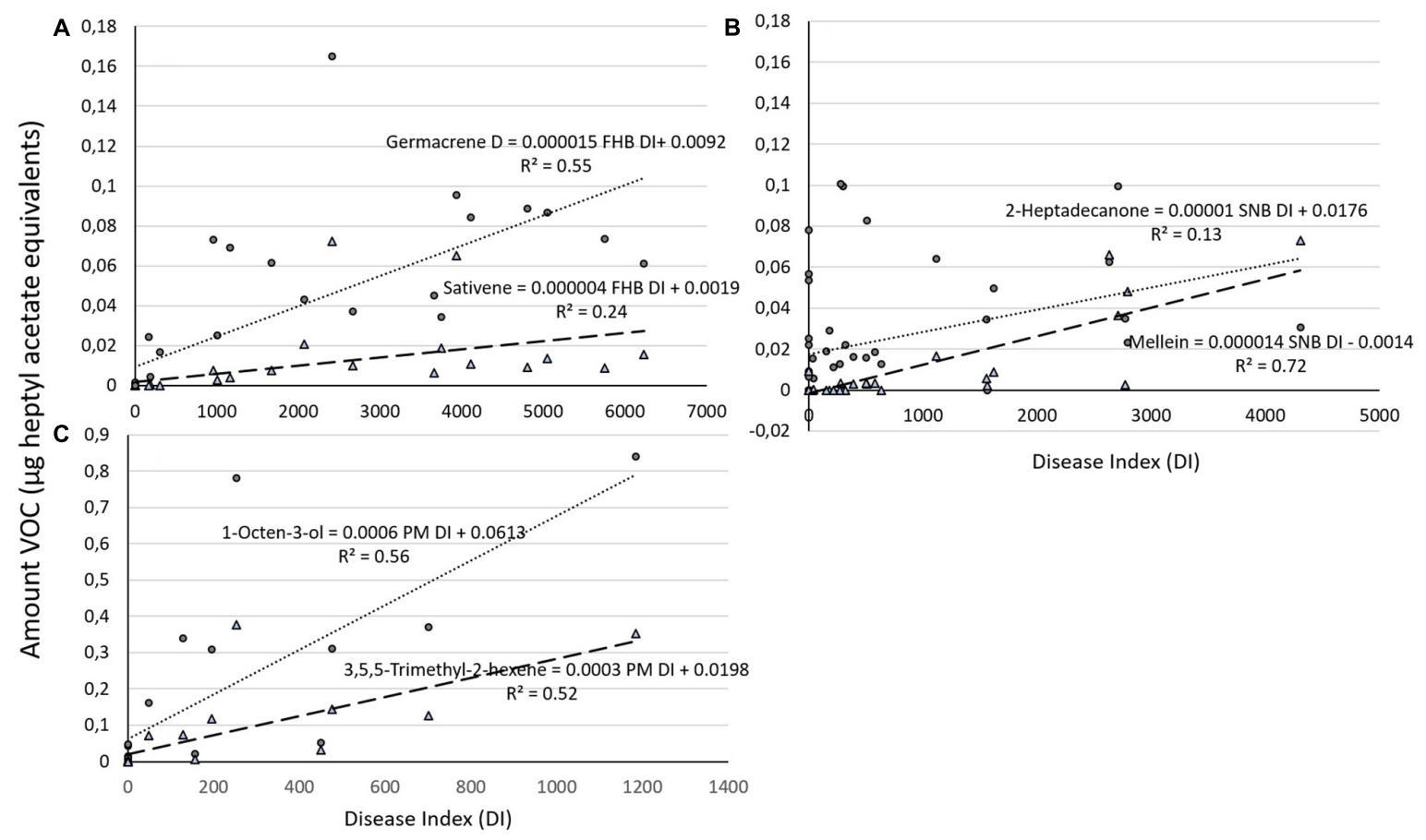

FIGURE 3 | Graphical representation of amount of (A) two different VOCs (germacrene and sativene) regressed against Fusarium head blight (FHB) DI on both varieties ('Zebra' and 'Bjarne') combined and of (B) two different VOCs (2-heptadecanone and mellein) regressed against Septoria nodorum blotch (SNB) DI on both varieties combined. (C) Graphical representation of the amount of 1-octen-3-ol and 3,5,5-trimethyl-2-hexene regressed against powdery mildew (PM) DI on 'Bjarne.' The regression equations and the $R^{2}$ value are placed next to the regression lines.

the relationship between germacrene $\mathrm{D}$ and FHB DI and between sativene and FHB DI remained significant ( $P=0.000$ and $P=0.002$, respectively). The $\mathrm{DI}$ for $\mathrm{FHB}$ explained $55 \%$ of variation in germacrene $\mathrm{D}$ (germacrene $\left.\mathrm{D}=0.0092+0.000015 \mathrm{FHB} \mathrm{DI}, R^{2}=0.55\right)$ and $24 \%$ of sativene emission (sativene $=0.0019+0.000004 \mathrm{FHB} \mathrm{DI}$, $R^{2}=0.24$; Figure 3A). SNB DI for both varieties combined was significantly correlated with mellein and 2-heptadecanone ( $P=0.001$ and $P=0.013$, respectively). The SNB DI still explained over $70 \%$ of variation in mellein emission in both 'Zebra' and 'Bjarne' combined (mullein $=-0.0014+0.000014$ SNB DI, $R^{2}=0.72$ ) and $13 \%$ of 2 -heptadecanone emission (2-heptadecanone $=0.0176+0.00001 \mathrm{SNB} \mathrm{DI}, R^{2}=$ 0.13 ; Figure 3B).

The relationships between the production of 1-octen-3ol and the DI for PM and 3,5,5-trimethyl-2-hexene and PM DI on 'Bjarne' were both significant $(P<0.001$ and $P=0.001$, respectively). The PM DI explained over $50 \%$ of the variation in 1-octen-3-ol (1-octen-3-ol $=0.0613+0.00061$ PM DI, $\left.R^{2}=0.56\right)$ and 3,5,5-trimethyl-2-hexene emission (3,5,5-trimethyl-2-hexene $=0.0198+0.0003 \mathrm{PM} \mathrm{DI}, R^{2}=$ 0.52 ; Figure 3C).

\section{DISCUSSION}

Our studies showed that different fungal species produced different VOC profiles and could be grouped into their respective isolate and disease group based on these profiles with a medium to high accuracy ( $84.2 \%$ for both). Isolates from the same species produced very similar VOC profiles. Our results are in agreement with studies on fungal species isolated from indoor environments that showed that VOC profiles are species and sometimes even strain specific (Kuske et al., 2005). However, several studies showed that the VOC profile can change with the substrate and the environmental conditions at which the fungal isolates are grown (Kuske et al., 2005). We used only one media to cultivate the fungal isolates, and differences in the species-specific VOC profile would be expected if we had used different growth media under varying light, temperature, and humidity conditions. The same fungal isolate that is producing a certain VOC blend on PDA under controlled conditions could produce a completely different VOC blend when grown on plants out in the field.

In our study, some, but not all, VOCs produced by the pathogens in pure culture on PDA were also produced in significant amounts when the same fungal isolate had infected its wheat host. While 2-non-anone; 1-octen-3-ol; and 3,5,5trimethyl-2-hexene were produced by $F$. graminearum isolate 201569 in pure culture, 'Zebra' and 'Bjarne' inoculated with this strain also emitted 3,5,5-trimethyl-2-hexene at 7, 14, and $21 \mathrm{dpi}$, but not always in amounts significantly different from the samples inoculated with other pathogens. At 14 and $21 \mathrm{dpi}$, 1-octen-3-ol was captured from FHB-inoculated plants, but it was only emitted in significantly different amounts by 'Zebra' at 14 dpi. Pure cultures of $P$. nodorum isolate 201254 emitted 2-nonanone; 1-octen-3-ol; 3,5,5-trimethyl-2-hexene, alpha-cedrene; 
germacrene D; $\alpha$-patcholene; and mellein, while 'Zebra' and 'Bjarne' inoculated with the same strain emitted non-significant amounts of 3,5,5-trimethyl-2-hexene at 7 and 14 dpi and alphacedrene only at 7 and $21 \mathrm{dpi}$. However, significant amounts of mellein were emitted by SNB isolate 201254 in pure culture on PDA and by 'Zebra' and 'Bjarne' plants at 14 and 21 dpi with the same SNB isolate, suggesting that this compound is produced directly by the fungus under various growth conditions. Mellein and its derivatives are phytotoxic compounds directly produced by $P$. nodorum via the polyketide pathway (Huff and Hamilton, 1979; Devys et al., 1992). The compound 2-heptadecanone, which was emitted at $7 \mathrm{dpi}$ of the two different wheat varieties inoculated with the SNB pathogen, but not by any of the fungal pathogens in pure culture, has been found in the essential oil of Warionia saharae (Znini et al., 2013) and other plant species, which might indicate that it is a general plant stress indicator rather than a specific pathogen indicator for SNB.

Germacrene D was emitted from 'Zebra' and 'Bjarne' plants inoculated with F. graminearum isolate 201569 at 7, 14, and 21 dpi. However, this strain did not consistently produce germacrene D in pure culture, and only F. graminearum isolate $140 / 80$ did emit this compound in significant amounts. In addition, $F$. poae and $P$. nodorum isolates 201254, 201252, and 202586 produced germacrene D in significant amounts. This could indicate that this particular VOC is produced under certain growth conditions by isolates of different fungal species and might be not specific and reliable enough to serve as a pathogenspecific indicator in the field. Germacrene A and C, but not $\mathrm{D}$, have been identified as metabolites in the terpene pathway of Fusarium fujikuroi (Burkhardt et al., 2016). Germacrene $\mathrm{D}$ is not unique to fungi, as it was also found in essential oils from several plant species (De Medeiros et al., 2016; Sampietro et al., 2016).

Sativene, on the other hand, was identified only from F. graminearum-inoculated plants but not from any of the Fusarium spp. isolates in pure culture. Flynn et al. (2019) reported that F. graminearum produces sativene when the fungus is grown in toxin induction medium, but not in non-induction medium. This suggests that sativene could be a pathogenicity factor in FHB, which might only be expressed in planta. As such, it would have the potential to serve as a pathogen-specific indicator for FHB in wheat if the amounts emitted would be sufficient for detection.

The VOC 2-(1-cyclopent-1-enyl-methylethyl)cyclopentanone associated with F. graminearum and F. poae in pure culture of our study has not been found in infected plants or emitted from fungi in any other study. Burdock (Arctium lappa) leaf essential oils contained 3,5,5-trimethyl-2-hexene, the compound found in 'Bjarne' inoculated with B. graminis f.sp. tritici, and was shown to inhibit Escherichia coli biofilms in earlier studies (Lou et al., 2013). This compound has not been described to be produced by fungi before. If it is commonly produced by wheat varieties infected with $B$. graminis f.sp. tritici, it could be a candidate as a pathogen-specific indicator for powdery mildew. The 2-non-anone is a methyl ketone found in many fruits and vegetables, wine, and beer. It was shown to be produced by $F$. poae on basic medium (van der Schaft et al., 1992), and it was contained in the VOC blend produced by the endophytic fungus Muscodor albus inhibiting P. nodorum (Strobel et al., 2001). However, it has not been associated with other wheat pathogenic fungi and has not been shown to be produced in pure culture by other Fusarium species or by P. nodorum. The compound 1-octen-3-ol was emitted in significant amounts by almost all isolates in pure culture, except for $F$. avenaceum 203587. It was also produced by 'Bjarne' infected with B. graminis f.sp. tritici in our study but interestingly not in 'Bjarne' or 'Zebra' when inoculated with FHB or SNB. This compound is known to be a major contributor to the characteristic mushroom aroma (Combet et al., 2006) and has been reported as a potential biomarker for B. graminis f.sp. tritici by Hamow et al. (2021). As B. graminis f.sp. tritici grows primarily on the surface of the plant, the characteristic mushroom smell might be emitted into the air by the superficial mycelium, while it might not be released from plants colonized by fungi growing in the interior of the plant. Both F. graminearum and $P$. nodorum grow inside their hosts, developing only pycnidia at the surface of the plant tissue that can emit any pathogenspecific VOC directly.

We demonstrated that VOC profiles of inoculated wheat plants were specific in the composition of the different VOCs emitted when infected with one pathogen at a time. There were differences in VOC profiles between wheat varieties infected with the same pathogen, but some compounds were emitted consistently from both varieties. Grouping the diseases based on a selection of the emitted VOCs over all sampling times leads to an overall accuracy of disease classification of $86 \%$ for 'Bjarne' and an overall accuracy of classification of $74 \%$ for 'Zebra.' Some compounds were emitted only during certain times after inoculation, but not during others, indicating that the timing of VOC collection is important for the actual detection of the pathogen.

The regression model based on the VOC profiles for both 'Zebra' and 'Bjarne' and DI of FHB, SNB, and PM showed that the relationship between VOC emission and disease intensity was variable, but significant. Increase in disease DI did not explain all the variations in specific VOC emission, supporting the idea that VOC blends or signatures are more useful in disease classification than single VOCs due to the many other factors influencing the emission of specific VOCs. However, the relationship between VOC emission and disease index could be useful to estimate the level of disease present based on the VOC concentration collected. There was no significant difference between the regression slope and the constant between the two wheat varieties, indicating that the relationship between VOC production and DI was robust across the two varieties tested. However, these models were developed based on plant inoculations under controlled greenhouse conditions, with only one single pathogen tested at a time, and cannot be expected to be transferrable to field conditions.

In our study, we collected VOC from wheat plants grown in the greenhouse and placed it in a closed container on a work bench in the laboratory for $24 \mathrm{~h}$. We have not explored the possible effect of daytime, light, temperature or humidity, 
or additional stress factors, such as other pathogens, physical damage, drought, or nutrient deficiency. Under field conditions, a multitude of diseases can attack a plant simultaneously. This might lead to the simultaneous production of disease-specific VOCs we have observed in our study or to the production of other not-yet-determined VOC blends.

Our studies showed that different diseases and fungal pathogens can be classified into their respective disease group based on specific VOC profiles from wheat plants under controlled conditions. We also showed that the amounts of VOCs are correlated with the disease index of the infected plants. It is the first step toward exploiting the VOC-based chemical communication system of crop plants to direct site-specific control measures for more effective pathogen control in cereal production systems. In order to further explore the role of VOCs as biomarkers for wheat diseases, we would need to collect VOCs from wheat grown in the field and include earlier sampling times and sample from plants under simultaneous attack of different isolates and diseases.

\section{DATA AVAILABILITY STATEMENT}

The original contributions presented in the study are included in the article/supplementary material, further inquiries can be directed to the corresponding author.

\section{REFERENCES}

Abel, C., Clauss, M., Schaub, A., Gershenzon, J., and Tholl, D. (2009). Floral and insect-induced volatile formation in Arabidopsis lyrata ssp. petraea, a perennial, outcrossing relative of A. thaliana. Planta 230, 1-11. doi: 10.1007/s00425-0090921-7

Algarra, T., Alarcon, A., Lazazzara, V., Cappellin, L., Bianchedi, P. L., Schuhmacher, R., et al. (2015). Emission of volatile sesquiterpenes and monoterpenes in grapevine genotypes following Plasmopara viticola inoculation in vitro. J. Mass Spectrom. 50, 1013-1022. doi: 10.1002/jms.3615

Ameye, M., Audenaert, K., De Zutter, N., Steppe, K., Van Meulebroek, L., Vanhaecke, L., et al. (2015). Priming of wheat with the green leaf volatile Z3-hexenyl acetate enhances defense against Fusarium graminearum but boosts deoxynivalenol production. Plant Physiol. 167, 1671-1684. doi: 10.1104/pp.15. 00107

Arimura, G. I., Shiojiri, K., and Karban, R. (2010). Acquired immunity to herbivory and allelopathy caused by airborne plant emissions. Photochemistry 71, 16421649. doi: 10.1016/j.phytochem.2010.06.021

Asalf, B., Gadoury, D. M., Tronsmo, A. M., Seem, R. C., Dobson, A., Peres, N. A., et al. (2014). Ontogenic resistance of leaves and fruit, and how leaf folding influences the distribution of powdery mildew on strawberry plants colonized by Podosphaera aphanis. Phytopathology 104, 954-963. doi: 10.1094/PHYTO12-13-0345-R

Asalf, B., Onofre, R. B., Gadoury, D. M., Peres, N. A., and Stensvand, A. (2021). Pulsed water mists for suppression of strawberry powdery mildew. Plant Dis. 105, 71-77. doi: 10.1094/PDIS-04-20-0735-RE

Bockus, W. W., Bowden, R. L., Hunger, R. M., Murray, T. D., and Smiley, R. W. (2010). Compendium of Wheat Diseases and Pests, 3rd Edn. Saint Paul, Min: American Phytopathological Society.

Brilli, F., Loreto, F., and Baccelli, I. (2019). Exploiting plant volatile organic compounds (VOCs) in agriculture to improve sustainable defense strategies and productivity of crops. Front. Plant Sci. 10:264. doi: 10.3389/fpls.2019.00264

Burkhardt, I., Siemon, T., Henrot, M., Studt, L., Rösler, S., Tudzynski, B., et al. (2016). Mechanistic characterisation of two sesquiterpene cyclases from the

\section{AUTHOR CONTRIBUTIONS}

AF designed and performed the experiments and wrote the manuscript. BA assisted with the experiments. HRN did the chemical analysis. All authors read the manuscript, gave editorial advice, and approved the submitted version.

\section{FUNDING}

This work was part of the project "Multisensory precision agriculture-improving yields and reducing environmental impact" (207829), financed by the Norwegian Research Council.

\section{ACKNOWLEDGMENTS}

We would like to thank Andrew Dobson for his extensive help in cultivating the plants in the green house and Geir Knudsen for his help with optimizing the collection of VOCs in the laboratory. We would like to thank Ingerd Skow Hofgaard for supplying us with the isolates from different Fusarium species and Jafar Razzaghian for producing single-spore isolates of the different pathogens tested. We are grateful to David Gadoury and Bob Seem for the helpful and critical discussions that led to the initiation of this project.

plant pathogenic fungus Fusarium fujikuroi. Angew. Chem. Int. Ed. Engl. 55, 8748-8751. doi: 10.1002/anie.201603782

Cardoza, Y. J., Alborn, H. T., and Tumlinson, J. H. (2002). In vivo volatile emissions from peanut plants induced by simultaneous fungal infection and insect damage. J. Chem. Ecol. 28, 161-174. doi: 10.1023/a:1013523104853

Combet, E., Henderson, J., Eastwood, D. C., and Burton, K. S. (2006). Eightcarbon volatiles in mushrooms and fungi: properties, analysis, and biosynthesis. Mycoscience 47, 317-326. doi: 10.1080/10408398.2017.1285269

Dalen, M., Knudsen, G. K., Norli, H. R., and Thöming, G. (2015). Sources of volatiles mediating host location behaviour of Glypta haesitator, a larval parasitoid of Cydia nigricana. Biol. Control 90, 128-140.

De Medeiros, F. C., Gouveia, F. N., Bizzo, H. R., Vieira, R. F., and Del Menezzi, C. H. (2016). Fungicidal activity of essential oils from Brazilian Cerrado species against wood decay fungi. Int. Biodeterior. Biodegrad. 114, 87-93.

Devys, M., Barbier, M., Bousquet, J. F., and Kollmann, A. (1992). Isolation of the New (-)-(3 R, 4S)-4-Hydroxymellein from the Fungus Septoria nodorum Berk. Z. Naturforsch. C 47, 779-781. doi: 10.1515/znc-1992-9-1024

Engle, J. S., Madden, L. V., and Lipps, P. E. (2003). Evaluation of inoculation methods to determine resistance reactions of wheat to Fusarium graminearum. Plant Dis. 87, 1530-1535. doi: 10.1094/PDIS.2003.87.12.1530

Fenske, M. P., and Imaizumi, T. (2016). Circadian rhythms in floral scent emission. Front. Plant Sci. 7:462. doi: 10.3389/fpls.2016.00462

Flynn, C. M., Broz, K., Jonkers, W., Schmidt-Dannert, C., and Kistler, H. C. (2019). Expression of the Fusarium graminearum terpenome and involvement of the endoplasmic reticulum-derived toxisome. Fungal Genet. Biol. 124, 78-87. doi: 10.1016/j.fgb.2019.01.006

Food and Agriculture Organization [FAO] (2019). Staple Foods: What Do People Eat? Available online at: http://www.fao.org/3/u8480e/u8480e07.htm (accessed July 25, 2019).

Gebbers, R., and Adamchuk, V. I. (2010). Precision agriculture and food security. Science 327, 828-831. doi: 10.1126/science.1183899

Gregg, P. C., Del Socorro, A. P., and Landolt, P. J. (2018). Advances in attractand-kill for agricultural pests: beyond pheromones. Annu. Rev. Entomol. 63, 453-470. doi: 10.1146/annurev-ento-031616-035040 
Guenther, A. (1997). Seasonal and spatial variations in natural volatile organic compound emissions. Ecol. Appl. 7, 34-45.

Hamow, K. Á, Ambrózy, Z., Puskás, K., Majláth, I., Cséplõ, M., Mátyus, R., et al. (2021). Emission of novel volatile biomarkers for wheat powdery mildew. Sci. Total Environ. 781:146767. doi: 10.1016/j.scitotenv.2021.146767

Huff, W. E., and Hamilton, P. B. (1979). Mycotoxins-their biosynthesis in fungi: ochratoxins-metabolites of combined pathways. J. Food Prot. 42, 815-820. doi: 10.4315/0362-028X-42.10.815

Jansen, R. M. C., Wildt, J., Kappers, I. F., Bouwmeester, H. J., Hofstee, J. W., and Van Henten, E. J. (2011). Detection of diseased plants by analysis of volatile organic compound emission. Annu. Rev. Phytopathol. 49, 157-174. doi: 10. 1146/annurev-phyto-072910-095227

Kim, J., and Felton, G. W. (2013). Priming of antiherbivore defensive responses in plants. J. Insect Sci. 20, 273-285. doi: 10.1111/j.1744-7917.2012.01584.x

Kuske, M., Romain, A. C., and Nicolas, J. (2005). Microbial volatile organic compounds as indicators of fungi. Can an electronic nose detect fungi in indoor environments? Build Environ. 40, 824-831.

Lin, M., Corsi, B., Ficke, A., Tan, K. C., Cockram, J., and Lillemo, M. (2020). Genetic mapping using a wheat multi-founder population reveals a locus on chromosome $2 \mathrm{~A}$ controlling resistance to both leaf and glume blotch caused by the necrotrophic fungal pathogen Parastagonospora nodorum. Theor. Appl. Genet. 133, 785-808. doi: 10.1007/s00122-019-03507-w

Lou, Z., Liu, Y., Hong, Y., Song, X., Wang, H., and Ai, L. (2013). Anti-biofilm activities and chemical composition of essential oil from burdock leaf. Food Sci. Technol. 19, 915-921.

Morath, S. U., Hung, R., and Bennett, J. W. (2012). Fungal volatile organic compounds: a review with emphasis on their biotechnological potential. Fungal Biol. Rev. 26, 73-83. doi: 10.1016/j.fbr.2012.07.001

Naznin, H. A., Kimura, M., Miyazawa, M., and Hyakumachi, M. (2013). Analysis of volatile organic compounds emitted by plant growth-promoting fungus Phoma sp. GS8-3 for growth promotion effects on tobacco. Microbes Environ. 28, 42-49. doi: 10.1264/jsme2.me12085

Naznin, H. A., Kiyohara, D., Kimura, M., Miyazawa, M., Shimizu, M., and Hyakumachi, M. (2014). Systemic resistance induced by volatile organic compounds emitted by plant growth-promoting fungi in Arabidopsis thaliana. PLoS One 9:e86882. doi: 10.1371/journal.pone.0086882

Oerke, E. C. (2006). Crop losses to pests. J. Agric. Sci. 144, 31-43. doi: 10.1017/ s0021859605005708

Pio, C. A., Silva, P. A., Cerqueira, M. A., and Nunes, T. V. (2005). Diurnal and seasonal emissions of volatile organic compounds from cork oak (Quercus suber) trees. Atmos. Environ. 39, 1817-1827. doi: 10.1016/j.atmosenv.2004.11. 018

Sampietro, D. A., Lizarraga, E. F., Ibatayev, Z. A., Omarova, A. B., Suleimen, Y. M., and Catalán, C. A. (2016). Chemical composition and antimicrobial activity of essential oils from Acantholippia deserticola, Artemisia proceriformis, Achillea micrantha and Libanotis buchtormensis against phytopathogenic bacteria and fungi. Nat. Prod. Res. 30, 1950-1955. doi: 10.1080/14786419.2015.1091453

Sankaran, S., Mishra, A., Ehsani, R., and Davis, C. (2010). A review of advanced techniques for detecting plant diseases. Comput. Electron. Agric. 72, 1-13. doi: 10.1016/j.compag.2010.02.007

Shulaev, V., Silverman, P., and Raskin, I. (1997). Airborne signalling by methyl salicylate in plant pathogen resistance. Nature 385:718. doi: 10.1038/385718a0

Steen, R., Norli, H. R., and Thöming, G. (2019). Volatile composition and timing of emissions in a moth-pollinated orchid in relation to hawkmoth (Lepidoptera:
Sphingidae) activity. Arthropod Plant Interact. 13, 581-592. doi: 10.1007/ s11829-019-09682-3

Stein, S. E. (1999). An integrated method for spectrum extraction and compound identification from gas chromatography/mass spectrometry data. J. Am. Soc. Mass Spectrom. 10, 770-781. doi: 10.1016/s1044-0305(99)00047-1

Stewart-Jones, A., and Poppy, G. M. (2006). Comparison of glass vessels and plastic bags for enclosing living plant parts for headspace analysis. J. Chem. Ecol. 32, 845-864. doi: 10.1007/s10886-006-9039-6

Strobel, G. A., Dirkse, E., Sears, J., and Markworth, C. (2001). Volatile antimicrobials from Muscodor albus, a novel endophytic fungus. Microbiology 147, 2943-2950. doi: 10.1099/00221287-147-11-2943

Tholl, D., Boland, W., Hansel, A., Loreto, F., Röse, U. S., and Schnitzler, J. P. (2006). Practical approaches to plant volatile analysis. Plant J. 45, 540-560. doi: 10.1111/j.1365-313X.2005.02612.x

Tholl, D., Hossain, O., Weinhold, A., Röse, U. S., and Wei, Q. (2021). Trends and applications in plant volatile sampling and analysis. Plant J. 106, 314-325. doi: $10.1111 /$ tpj.15176

Thompson, C. H., McCartney, M. M., Roubtsova, T. V., Kasuga, T., Ebeler, S. E. Davis, C., et al. (2021). Analysis of volatile profiles for tracking asymptomatic infections of Phytophthora ramorum and other pathogens in Rhododendron. Phytopathology 111, 1818-1827. doi: 10.1094/PHYTO-10-20-0472-R

Tikunov, Y., Lommen, A., De Vos, C. R., Verhoeven, H. A., Bino, R. J., Hall, R. D., et al. (2005). A novel approach for nontargeted data analysis for metabolomics. Large-scale profiling of tomato fruit volatiles. Plant Physiol. 139, 1125-1137. doi: 10.1104/pp.105.068130

van der Schaft, P. H., ter Burg, N., van den Bosch, S., and Cohen, A. M. (1992). Fedbatch production of 2-heptanone by Fusarium poae. Appl. Microbiol. Biotech. 36, 709-711. doi: 10.1007/bf00172179

Witzgall, P., Kirsch, P., and Cork, A. (2010). Sex pheromones and their impact on pest management. J. Chem. Ecol. 36, 80-100. doi: 10.1007/s10886009-9737-y

Zadoks, J. C., and Schein, R. D. (1979). Epidemiology and Plant Disease Management. Wageningen: Agricultural University.

Znini, M., Cristofari, G., Majidi, L., El Harrak, A., Paolini, J., and Costa, J. (2013). In vitro antifungal activity and chemical composition of Warionia saharae essential oil against 3 apple phytopathogenic fungi. Food Sci. Biotech. 22, 113-119. doi: 10.1007/s10068-013-0056-2

Conflict of Interest: The authors declare that the research was conducted in the absence of any commercial or financial relationships that could be construed as a potential conflict of interest.

Publisher's Note: All claims expressed in this article are solely those of the authors and do not necessarily represent those of their affiliated organizations, or those of the publisher, the editors and the reviewers. Any product that may be evaluated in this article, or claim that may be made by its manufacturer, is not guaranteed or endorsed by the publisher.

Copyright (C) 2022 Ficke, Asalf and Norli. This is an open-access article distributed under the terms of the Creative Commons Attribution License (CC BY). The use, distribution or reproduction in other forums is permitted, provided the original author(s) and the copyright owner(s) are credited and that the original publication in this journal is cited, in accordance with accepted academic practice. No use, distribution or reproduction is permitted which does not comply with these terms. 